\title{
RAREFIED PURE GAS TRANSPORT IN NON-ISOTHERMAL POROUS MEDIA : VALIDATION AND TESTS OF THE MODEL
}

\author{
Gérard L. Vignoles ${ }^{1}$ (vinhola@lcts.u-bordeaux1.fr) \\ 1. Université Bordeaux l, \\ Lab. for ThermoStructural Composites (LCTS), \\ UMR 5801 CNRS-CEA-Safran-UBl, \\ 3. Allée La Boëtie, \\ F-33600 Pessac, France
}

Pierre Charrier $^{2}$ (pierre.charrier@math.u-bordeaux1.fr)

, Christophe Preux ${ }^{2,3}$ (christophe.preux@math.u-bordeaux1.fr)

and Bruno Dubroca ${ }^{2,3}$ (bruno.dubroca@math.u-bordeaux1.fr)

2. Université Bordeaux l,

Applied Mathematics of Bordeaux (MAB),

UMR 5466 CNRS-UB1, LRC-CEA M03,

351 Cours de la Libération,

F-33405 Talence Cedex, France

3. Commissariat à l'Energie Atomique (CEA)

Centre d'Etudes Scientifiques et Techniques d'Aquitaine, BP2, F-33114 Le Barp, France

\begin{abstract}
Viscous flow, effusion, and thermal transpiration are the main gas transport modalities for a rarefied gas in a macro-porous medium. They have been well quantified only in the case of simple geometries. This paper presents a numerical method based on the homogenization of kinetic equations producing effective transport properties (permeability, Knudsen diffusivity, thermal transpiration ratio) in any porous medium sample, as described $e$. g.by a digitized 3D image. The homogenization procedure - neglecting the effect of gas density gradients on heat transfer through the solid - leads to closure problems in $\mathbb{R}^{6}$ for the obtention of effective properties ; they are then simplified using a Galerkin method based on a 21-element basis set. The kinetic equations are then discretized in $\mathbb{R}^{3}$ space with a finite-volume scheme. The method is validated against experimental data in the case of a closed test tube. It shows to be coherent with past approaches of thermal transpiration. Then, it is applied to several 3D images of increasing complexity. Another validation is brought by comparison with other distinct numerical approaches for the evaluation of the Darcian permeability tensor and of the Knudsen diffusion tensor. Results show that thermal transpiration has to be described by an effective transport tensor which is distinct from the other tensors.
\end{abstract}

Keywords: Knudsen diffusion, thermal transpiration, kinetic equation, homogenization, numerical methods 


\section{List of symbols}

\section{Latin}

- $A$ : Matrix element in numerical problem

- $b_{0}, \mathbf{b}_{1}, b_{2}$ : Expansion parameters for the closure variable $\alpha$

- $B$ : Porous medium intrinsic permeability $\left(m^{2}\right)$

- c : Microscopic molecular velocity

- $\bar{c}$ : Mean quadratic velocity $\left(m . s^{-1}\right)$

- $C_{v}$ : Specific heat capacity at constant volume $\left(J . \mathrm{kg}^{-1} \cdot \mathrm{K}^{-1}\right)$

- $d_{0}, \mathbf{d}_{1}, d_{2}$ : Expansion parameters for the closure variable $\beta$

- $\partial Y_{f s}$ : Interface between domains $Y_{f}$ and $Y_{s}$

- $D_{K}:$ Knudsen diffusion coefficient $\left(m^{2} \cdot s^{-1}\right)$

- $\underline{\underline{D}}$ : Effective tensor linking mass flux and density gradient $\left(m^{2} \cdot s^{-1}\right)$

- $\underline{\underline{D}}$ : Effective tensor linking mass flux and temperature gradient $\left.\overline{\overline{(k}} g \cdot m^{-1} \cdot s^{-1} \cdot K^{-1}\right)$

- $d_{p}$ : Pore diameter $(m)$

- $E$ : Internal (translational) energy $(J)$

- $f$ : Mass distribution function in space and velocity spaces $\left.\left(k g \cdot m^{-9} \cdot s^{3}\right)\right)$

- $g$ : Second member in numerical problem

- $\underline{\underline{K}}$ : Effective heat conductivity tensor $\left(W \cdot m^{-1} \cdot K^{-1}\right)$

- $K$ : Auxiliary matrix in numerical problem

- $k_{B}$ : Boltzmann's constant $\left(J . K^{-1}\right)$

- Kn: Knudsen number (-)

- $\bar{l}$ : Mean free path $(m)$

- $L$ : Reference length $(m)$

- $L(f)$ : Linearized collision operator $\left(k g \cdot m^{-9} \cdot s^{-4}\right)$ 
- $\quad M$ : Molecular mass $\left(k g \cdot \mathrm{mol}^{-1}\right)$

- $M_{n}(f)$ : Maxwellian distribution normalized to unity for half-flux $\left(m^{-4} \cdot s^{4}\right)$

- Ma: Mach number (-)

- $m_{j}$ : Functions of the basis set (various units)

- $\quad N$ : Total number of basis functions

- $\mathbf{n}$ : Normal vector (-)

- $P$ : Pressure $(P a)$

- $Q(f, f)$ : Collision operator $\left(k g \cdot m^{-9} \cdot s^{2}\right)$

$-\mathcal{R}=\mathcal{N}_{A} k_{B}:$ Perfect gas constant $\left(J \cdot \mathrm{mol}^{-1} K^{-1}\right)$

- Re : Reynolds number (-)

$-\quad S$ : Numerical coefficient matrix

- $T:$ Temperature $(K)$

$-T_{c}:$ Critical temperature $(K)$

$-t:$ Time $(s)$

- $\mathbf{u}$ : Intrinsic mass-average gas velocity $\left(\mathrm{m} . \mathrm{s}^{-1}\right)$

- $\mathbf{v}$ : Extrinsic mass-average gas velocity $\left(m . s^{-1}\right)$

- $\mathbf{w}$ : Mute integration variable for molecular velocity $\left(\mathrm{m} . \mathrm{s}^{-1}\right)$

- $\mathbf{x}$ : Space coordinates $(m)$

- $\mathbf{x}^{\prime}$ : Large-scale space coordinate $(m)$

- $\mathbf{y}$ : Small-scale space coordinate $(m)$

- $Y$ : Small space subset

\section{Greek}

- $\alpha$ : Closure variable $\left(m . K^{-1}\right)$

- $\alpha_{T}$ : Thermal diffusion ratio (-) 
- $\beta$ : Closure variable $\left(m^{4} \cdot k g^{-1}\right)$

- $\gamma$ : Closure variable $(m)$

- $\epsilon$ : Porosity (-)

- $\varepsilon$ : Small parameter for asymptotics (-)

- $\eta$ : Gas viscosity (Pa.s)

- $\theta_{j}$ : Auxiliary basis functions

- $\lambda$ : Thermal conductivity $\left(W \cdot m^{-1} \cdot K^{-1}\right)$

- $\nu$ : Kinematic viscosity $\left(m^{2} \cdot s^{-1}\right)$

- $\xi$ : Correction factor accounting for non-specular collisions (-)

- $\pi_{g}, \pi_{g}^{\prime}$ : Pressure correction parameters for Knudsen diffusion $(\mathrm{Pa})$

- $\rho:$ Gas density $\left(k g \cdot m^{-3}\right)$

- $\sigma$ : Accommodation factor (-)

- $\sigma_{c}$ : Collision diameter $(m)$

$-\Sigma:$ Fluid/solid interface

- $\tau$ : Relaxation time $(s)$

- $\underline{\underline{\tau}}:$ Tortuosity (-)

- $\quad \psi$ : Any function in $\mathbb{R}_{c}^{3}$ velocity space (-)

- $\omega$ : Orientation $(s r)$

- $\Omega$ : Open set of $\mathbb{R}^{3}$ position space

- $\Omega_{v}$ : Collision integral for gas viscosity (-)

\section{Subscripts and underscripts}

- $\cdot$ : vector in $1, \mathbf{c},|c|^{2}$ space

- $[\cdot]_{1}$ : first-order approximation in DGM derivation

$-{ }_{c}$ : relative to gas critical point 
$-\cdot_{d}$ : relative to dust

- $\cdot_{f}$ : relative to fluid phase

- $\cdot_{g}$ : relative to gas species

$-\cdot_{K}$ : relative to Knudsen transport

- $\cdot_{p}$ : relative to pore $(\mathrm{s})$

$-\cdot_{s}$ : relative to solid phase

- $\cdot_{t r}$ : translational contribution

- ${ }_{v}$ : relative to viscous transport

$-\cdot_{*}$ : reference quantity

\section{Introduction}

Thermostructural composites are high performance materials aimed at very special applications in aerospace engineering, like thermal protection systems for atmospheric re-entry, aeroplane brakes, rocket nozzles and jet reactor parts. They are constituted by a network of fibers - carbon or ceramic - surrounded by a matrix - also carbon or ceramic, with possible insertion of an interphase between them (Naslain, 1985) : such a structure brings them a combination of the strength from the constituents, and additional toughness when the interphase is correctly chosen. This is a great advantage, but the drawbacks are the complexity of the processing technique and of the composite's behavior, not only mechanical, but also thermal and physico-chemical.

One of the issues in ceramic-matrix composites (CMC) or carbon/carbon composites (CCC) fabrication is the precise knowledge of the ability that an arrangement of fibers (the preform) has to let itself be infiltrated by gaseous precursors of the matrix, resulting in the densest possible material (Ofori and Sotirchos, 1996b). Chemical Vapor Infiltration (CVI) is the generic name of the gas-phase route processes that are currently employed (Naslain and Langlais, 1990; Besmann et al., 1991); many configurations make use of strong thermal gradients in order to monitor better the localization of the chemical deposition reaction inside the porous medium (Vignoles et al., 
2005; Vignoles et al., 2006). Low pressures are also common in CVI, since it is a convenient way to reduce the Thiele modulus ; accordingly, the Knudsen number is usually not negligible. A large amount of modelling efforts have been made (Starr and Smith, 1990; Ofori and Sotirchos, 1996a; Reuge and Vignoles, 2005; Vignoles, 2006), featuring or not Knudsen transport, but few has been said about thermal transpiration in these works.

Another key point in thermostructural composites is the knowledge of their physico-chemical behavior in conditions of utilization, which usually are extremely severe : high temperatures, presence of aggressive gases, strong thermal gradients, etc ... The material is rapidly very different from the as-fabricated state and begins to display cracks and fissures. One of the most dramatic examples is the carbon/phenolic resin composite used in atmospheric re-entry : the matrix is not a high-temperature material in itself, but its pyrolysis has two advantages : $i$ ) it consumes heat, and $i i$ ) it yields a porous carbon material, which has non-negligible thermostructural properties. The pyrolysis occurs in presence of extreme thermal gradients. Again, taking into account the (sub-)micrometer size of the pores, the Knudsen number is potentially large. This is another motivation for the study of rarefied gas transport in a porous medium in the presence of thermal gradients.

Whatever the application case, the geometry of the studied media is far from being simple. In order to be able to provide accurate estimations of the transport parameter in arbitrarily complex 3D porous media, we have derived a formulation for pure gas transport (that is, not including binary diffusion) in non-isothermal conditions as it appears from homogenization theory (Bensoussan et al., 1978; Bardos et al., 1997) when applied to the Maxwell-Boltzmann kinetic equation. The associated energy transport is also treated. The performed change of scale provides a set of macroscopic variables and equations, as well as a set of closure problems that are to be solved at microscopic scale (Vignoles et al., 2008).

The purpose of the present work is to design a tool allowing to compute effective properties for intermediate and Knudsen transport, and for thermal transpiration in whatever porous medium.

A test of the approach is then given by modelling the thermal transpiration experiments of Malinauskas et al (Malinauskas et al., 1970). The numerical results will be discussed with respect to macroscopic models, such as the DGM and BFM theories, which will be briefly 
recalled in the pure gas case in the next section, and to kinetic-theory level existing results. Finally, computations performed in porous media differing from straight pores will be presented and discussed.

\section{Brief presentation of the homogenization model}

The identification of transport coefficients, and in particular of the precise impact of the pore geometries, has been treated mostly in isothermal conditions. The limiting cases of pure viscous flow (i.e. Darcy regime), pure diffusive flow, and pure Knudsen flow have been often separately addressed. Other works have considered the transition between two of the three transport modalities, mainly binary and Knudsen diffusion, by means of random walk algorithms in idealized 3D images of porous media (Burganos and Sotirchos, 1989; Tomadakis and Sotirchos, 1991) or by variational methods applied to media considered through their $n$-point correlation distributions (Strieder and Prager, 1968; Strieder, 1971). The consideration of slip-flow in addition to viscous flow in a change-of-scale methodology has been formally presented in (Whitaker, 1987). Homogenization performed on the same problem has been dealt with in (Skjetne and Auriault, 1999).

Thermal transpiration has been studied by numerous authors on the basis of kinetic equations, but almost always in the geometry of a straight cylindrical tube. See e.g. a short review in (Sharipov, 1996). It has already been shown, in such geometries, that working directly with the "Bhatnagar-Gross-Krook" (BGK) approximation of the kinetic equations (Loyalka, 1969) gives results for the "thermomolecular pressure difference" which are not too far from the DustyGas Model predictions (Gupta and Storvick, 1970). Direct Simulation by Monte-Carlo (DSMC) methods are also successful in describing rarefied gas dynamics in non-isothermal media ; up to now, most of the reported applications refer to rather simple geometries. Moreover, direct simulation does not enter straightforwardly in the frame of a change-of-scale strategy. So, to the author's knowledge, no method had been reported for the precise numerical evaluation of thermal transpiration parameters in the rarefied regime for general porous media described by a 3D image. This is the main purpose of the present work. 
The full derivation of the model is discussed in (Vignoles et al., 2008) and (Charrier and Dubroca, 2003). Here the main steps of the procedure will be recalled. First, the local kinetic problem is presented ; then, an analysis of the space decouplings and scalings is performed ; then, the homogenization is performed, and the final form of the homogenized equations, as well as the associated closure problems are obtained.

\subsection{LOCAL PROBLEM FORMULATION}

Let us denote $\Omega$, the open set in $\mathbb{R}^{3}$ occupied by the porous material, $\Omega_{s} \subset \Omega$ the open subset of $\Omega$ occupied by the solid phase, $\Omega_{f}$ the open subset occupied by the gas and $\Sigma$ the interface between $\Omega_{f}$ et $\Omega_{s}$. In all the following $\mathbf{n}=\mathbf{n}(\mathbf{x}), \mathbf{x} \in \Sigma$, denotes the normal to $\Sigma$ at point $\mathbf{x}$, outgoing from $\Omega_{s}$. The solid is assumed to have constant properties ; the gas will be described at the kinetic level, introducing the mass distribution function $f=f(\mathbf{x}, t, \mathbf{c})$. The usual macroscopic quantities are obtained by application of the velocity space averaging operator $<\bullet>=\int_{\mathbb{R}_{c}^{3}} \bullet \mathrm{d} \mathbf{c}$ :

$$
\rho=<f>, \rho \mathbf{u}=<f \mathbf{c}>, \rho E=<f \frac{|c|^{2}}{2}>,
$$

Under this set of assumptions, the local problem to solve is :

$$
\begin{aligned}
\partial_{t} f+\mathbf{c} \cdot \nabla_{x} f & =Q(f, f), \mathbf{x} \in \Omega_{f}, \\
\rho c_{v} \partial_{t} T & =\left(\operatorname{div}_{x} k_{s} \nabla_{x} T\right), \mathbf{x} \in \Omega_{s}, \\
f(\mathbf{x}, \mathbf{c})_{\mid \mathbf{c} \cdot \mathbf{n}>0} & =\sigma\left(\int_{\mathbf{w} \cdot \mathbf{n}<0} \mathbf{w} \cdot \mathbf{n} \mid f(\mathbf{x}, \mathbf{w}) \mathrm{d} \mathbf{w}\right) M_{n}(T) \\
+(1-\sigma) & f(\mathbf{x}, \mathbf{c}-2 \mathbf{c} \cdot \mathbf{n} \mathbf{n}), \mathbf{x} \in \Sigma, \\
k_{s} \nabla_{x} T \cdot \mathbf{n} & =-\int \frac{|c|^{2}}{2} f(\mathbf{x}, \mathbf{c}) \mathbf{c} \cdot \mathbf{n d} \mathbf{c}, \mathbf{x} \in \Sigma,
\end{aligned}
$$

where we have introduced the collision operator $Q(f, f)$ and a normalized Maxwellian distribution :

$$
M_{n}(T)(\mathbf{c})=\frac{1}{2 \pi(\mathcal{R} T)^{2}} \exp \left(-\frac{|c|^{2}}{2 \mathcal{R} T}\right)
$$

The collision operator $Q(f, f)$ is approximated in this work by the Ellipsoidal-Statistical model (Holway, 1966). This model has the property of giving the correct Prandtl number (Andries et al., 2000); 
this is why it is expected to behave better than the simpler BhatnagarGross-Krook (Bhatnagar et al., 1954), especially in non-isothermal problems.

Such a model obeys the following properties :

- Global conservation of mass and energy are ensured

- The H-theorem is verified, that is, the local entropy production is positive.

\subsection{Space Decoupling and SCAlings}

It will now be assumed that the space variable may decoupled into two contributions, differing by their scale :

$$
\mathbf{x}=\mathbf{x}^{\prime}+\mathbf{y}
$$

where $\mathbf{x}^{\prime}$ has broad variations (i.e. $\psi\left(\mathbf{x}^{\prime}\right)$ is a local average of $\left.\psi(\mathbf{x})\right)$ and $\mathbf{y}$ has short-scale variations (i.e. $\psi(\mathbf{y})$ is a local perturbation of $\psi(\mathbf{x})$ with respect to $\left.\psi\left(\mathbf{x}^{\prime}\right)\right)$. Let us define an integration support $Y \in \Omega$ for $\mathbf{y}$, which is a small space region, split into two subsets $Y_{s}$ and $Y_{f}$. The ratio $\varepsilon=y / x$ is a small parameter allowing to use the tools of asymptotic analysis. By taking the limit $\varepsilon \rightarrow 0$, one simultaneously changes scale in space and takes a hydrodynamic limit for the fluid.

It is here considered that the fluid and the solid are in local equilibrium, so that only one temperature defined on $\Omega$ is enough to describe the system. Choosing a reference length $L=\mathcal{O}\left(|\Omega|^{1 / 3}\right)$ brings a small parameter $\varepsilon=d_{p} / L$. The system (2-5) is recast into a dimensionless form, and the identified scalings are :

- $M a \approx \varepsilon$, i.e., the flow is subsonic,

- $K n \approx \varepsilon$, i.e. the gas is in a transition regime. It will be practical to design a pore Knudsen number $K n_{p}=\varepsilon^{-1} K n$.

- As a consequence, $R e=\frac{\gamma M}{K n}=\mathcal{O}(1)$, so the flow is in a laminar regime.

- In the considered applications (temperatures around $1000 K$ and pressures from 1 to $100 \mathrm{kPa}$ ), the thermal conductivity ratio $\frac{\lambda_{g}}{\lambda_{s}}$ is small, and scales as $\varepsilon$. 
- Since the density ratio $\frac{\rho_{g}}{\rho s}$ also scales as $\varepsilon$ and the mass heat capacity ratio is of order unity, the thermal diffusivity ratio is expected to be unity.

- There is a weak coupling between the gas and the solid.

The scaled equations may be now rewritten as :

$$
\begin{aligned}
\varepsilon \partial_{t} f+\mathbf{c} \cdot \nabla_{x} f & =\frac{1}{\varepsilon} Q(f, f), \mathbf{x} \in \Omega_{f}, \\
\rho c_{v} \partial_{t} T & =\operatorname{div}_{x}\left(\lambda_{s} \nabla_{x} T\right), \mathbf{x} \in \Omega_{s}, \\
f_{\mid \mathbf{c} \cdot \mathbf{n}>0} & =\sigma\left(\int_{\mathbf{w} \cdot \mathbf{n}<0}|\mathbf{w} \cdot \mathbf{n}| f \mathrm{~d} \mathbf{w}\right) M_{n}(T) \\
+(1-\sigma) & f(\mathbf{x}, \mathbf{c}-2 \mathbf{c} \cdot \mathbf{n} \mathbf{n}), \mathbf{x} \in \Sigma, \\
\lambda_{s} \nabla_{x} T \cdot \mathbf{n} & =-\int \frac{|c|^{2}}{2} f \mathbf{c} \cdot \mathbf{n} \mathrm{d} \mathbf{c}, \mathbf{x} \in \Sigma,
\end{aligned}
$$

Note that the quantities $f, \mathbf{c}, \mathbf{x}, \rho C_{v}, T, \lambda_{s}, M_{n}, Q$ are now dimensionless.

\subsection{Homogenization}

Expanding $f$ and $T$ in successive powers of $\varepsilon$ yields a series of relations, that need to be closed in order to give useful information. An important result is that $f_{0}$, the zeroth-order approximation of $f$, is an absolute Maxwellian distribution, independent of $\mathbf{y}$, with temperature $T_{0}$; its average over velocity space is the zeroth-order approximation for the density $\rho_{0}$. Thus, $\nabla_{x^{\prime}} f_{0} / f_{0}$ can be written as a linear combination of $\nabla_{x^{\prime}} T_{0}$ and $\nabla_{x^{\prime}} \rho_{0}$.

On the other hand, the coupling between $T$ and $f$ is weak. Physically, this means that the solid phase, being much more conductive than the fluid phase with respect to heat, ensures by itself the buildup of the thermal gradient, which is then communicated to the fluid. So there is no effect of $\rho$ on $T$ to be taken into account.

It is then justified to seek $f_{1}$ and $T_{1}$ in the form :

$$
\begin{aligned}
& f_{1}=-f_{0}\left(\alpha \cdot \nabla_{x^{\prime}} T_{0}+\beta \cdot \nabla_{x^{\prime}} \rho_{0}\right) \\
& T_{1}=\gamma \cdot \nabla_{x^{\prime}} T_{0}
\end{aligned}
$$

The vectorial closure variables $\alpha=\left(\alpha_{i}(\mathbf{y}, \mathbf{c})\right)_{i=1,2,3}, \beta=\left(\beta_{i}(\mathbf{y}, \mathbf{c})\right)_{i=1,2,3}$, and $\gamma=\left(\gamma_{i}(\mathbf{y}, \mathbf{c})\right)_{i=1,2,3}$ are solutions of auxiliary problems defined in the elementary cell $Y$ : 


$$
\begin{gathered}
\left\{\begin{array}{l}
-L\left(f_{0} \alpha_{i}\right)+f_{0} \mathbf{c} \cdot \nabla_{y} \alpha_{i}=-f_{0} c_{i}\left(-\frac{3}{2 T_{0}}+\frac{M|c|^{2}}{2 \mathcal{R} T_{0}^{2}}\right), \text { in } Y_{f} \\
\alpha_{i}-\sigma\left(-\frac{2}{T_{0}}+\frac{M|c|^{2}}{2 \mathcal{R} T_{0}^{2}}\right) \gamma_{i}=\sigma \int_{\mathbf{w} \cdot \mathbf{n}<0}|\mathbf{n}| \alpha_{i} M_{n}\left(T_{0}\right) d \mathbf{w} \ldots \\
\ldots+(1-\sigma) \alpha_{i}(\mathbf{y}, \mathbf{c}-2 \mathbf{c} \cdot \mathbf{n} \mathbf{n}), \text { in } \partial Y_{f s}, \text { and } \forall \mathbf{c} \cdot \mathbf{n}>0
\end{array}\right. \\
\left\{\begin{array}{l}
-L\left(f_{0} \beta_{i}\right)+\mathbf{c} \cdot \nabla_{y}\left(f_{0} \beta_{i}\right)=-\frac{f_{0}}{\rho_{0}} c_{i}, \text { in } Y_{f} \\
\beta_{i}=\sigma \int_{\mathbf{w} \cdot \mathbf{n}<0} \cdot \mathbf{n} \mid \beta_{i} M_{n}\left(T_{0}\right) d \mathbf{w} \ldots, \beta_{i}(\mathbf{y}, \mathbf{c}-2 \mathbf{c} \cdot \mathbf{n} \mathbf{n}), \text { in } \partial Y_{f s}, \text { and } \forall \mathbf{c} \cdot \mathbf{n}>0 \\
\ldots+(1-\sigma), \text { in } Y_{f}
\end{array}\right. \\
\left\{\begin{array}{c}
-\operatorname{div}_{y}\left(\lambda_{s} \nabla_{y} \gamma_{i}\right)=0 \quad \text { in } \partial Y_{f s} \\
\lambda_{s} \nabla_{y} \gamma_{i} \cdot \mathbf{n}=-\lambda_{s} n_{i}, \text { in }
\end{array}\right.
\end{gathered}
$$

On a macroscopic scale, a description is sought only for the macroscopic variables; accordingly, one has to simultaneously integrate in velocity space and perform an average on the local support $Y$. The asymptotic transport model takes then the following form :

$$
\begin{aligned}
\partial_{t} \rho_{0}-\operatorname{div}_{x}\left(\underline{\underline{D}} \nabla_{x} \rho_{0}\right)-\operatorname{div}_{x}\left(\underline{\underline{\underline{D}}} \nabla_{x} T_{0}\right) & =0 \\
\rho_{s} C_{v} \partial_{t} T_{0}-\operatorname{div}_{x}\left(\underline{\underline{K}} \nabla_{x} T_{0}\right) & =0
\end{aligned}
$$

where the tensorial effective coefficients are built with the closure variables obtained from problems $(14,15,16)$ in the following way :

$$
\begin{gathered}
\underline{\underline{D}}=\left\langle-f_{0} \mathbf{c} \otimes[\beta]_{Y_{f}}\right\rangle \\
\underline{\underline{\tilde{D}}}=\left\langle-f_{0} \mathbf{c} \otimes[\alpha]_{Y_{f}}\right\rangle \\
\underline{\underline{K}}=\left[\lambda_{s}\left(\underline{\underline{\mathrm{Id}}}+\nabla_{y} \gamma\right)\right]_{Y_{s}}
\end{gathered}
$$

Here angular brackets still denote velocity-space averaging, and the square brackets denote the extrinsic coordinate-space averaging : $[\psi]_{Y_{i}}=\frac{1}{|Y|} \int_{Y_{i}} \psi(\mathbf{y}) d \mathbf{y}$. The two averaging procedures are intimately mixed since the gas is not in continuum regime inside the pores, so 
there is an implicit need of a space region of size $|Y|$ in order to perform a velocity-space averaging in a short enough time interval.

\section{Tests on thermal transpiration}

The goal of this section is to present a validation of the proposed model with physical data, namely the straight-pore results for monatomic gases reported by Malinauskas et al. (1970).

\subsection{Test Case presentation}

A bundle of ten 0.2- $\mathrm{mm}$ inner diameter, $76-\mathrm{mm}$ length Pyrex glass capillaries was the test porous medium. The extremities were held in chambers where the temperature was carefully monitored $\left(T_{1}=\right.$ $335.6 \mathrm{~K}$ at the bottom end, and $T_{2}=569.8 \mathrm{~K}$ at the top end), and the pressure difference between the two extremities $\left(\Delta P=P_{1}-P_{2}\right)$ was recorded as a function of the absolute pressure at the top, hottest side $\left(P_{2}\right)$. The DGM model equations :

$$
\left\{\begin{aligned}
\mathbf{v}=\epsilon \mathbf{u} & =-\left(\frac{P B}{\eta}+D_{K}\right) \frac{\nabla P}{P}-\alpha_{T} D_{K} \frac{\nabla T}{T} \\
D_{K} & =\frac{\epsilon}{3} \bar{c} d_{p} \underline{\underline{\tau_{K}}}-\frac{P+\pi_{g}}{P+\pi_{g}^{\prime}} \xi \\
\xi & =1+\frac{\frac{\pi}{8} \sigma}{B}=\underline{\underline{\epsilon \tau_{v}}}{ }^{-1} \frac{d_{p}^{2}}{32}
\end{aligned}\right.
$$

have been fitted to the curves $\Delta P=f\left(P_{2}\right)$, first by deriving an approximate estimation of $\Delta P$ involving a reference temperature $R_{m}=\sqrt{T_{1} T_{2}}$, and treating parameters $\alpha_{L}=\lim _{K n \rightarrow \infty} \alpha_{T}, \pi_{g}$ and $\pi_{g}^{\prime}$ as adjustable. We will show that an integration of the presented model leads to a correct agreement with the experimental data without parameter fitting, as was already shown by Storvick et al. (Storvick et al., 1978) with a BGK-model specialized to single-straight-pore computations.

\subsection{Numerical Procedure}

In the following, we will present the numerical procedure that has been developed for the use of the change-of-scale model, which is split into 
two parts : first, the estimation of "local" coefficients $D$ and $\tilde{D}$, that is, for any fixed value of $(P, T)$ of interest (i.e. in the range $\left[P_{1}, P_{2}\right] \times$ $\left.\left[T_{1}, T_{2}\right]\right)$; then, an integration of the balance equations from one side to the other of the capillaries, taking into account their nonlinear character. We will drop the tensorial notations in this section, because transport occurs only in one single direction.

\subsubsection{Extraction of the effective coefficients}

The method that has been used for the solution of eqs. $(14,15)$ is based on the projection of the kinetic-like equations on a basis set of functions $\left(m_{j}\right)_{j=1, N}$ through a Galerkin method on c. This is a generalization of a "moment method", since the basis set is not necessarily polynomial, as it will be seen later. Let us focus for example on problem (15). The unknowns $\beta_{i}$ are sought as a $\operatorname{sum} \sum_{j=1}^{N} \beta_{i}^{j}(\mathbf{y}) m_{j}(\mathbf{c})$, so one gets :

$$
\forall k \in\{1, N\} \quad \sum_{j=1}^{N}\left(-\beta_{i} L\left(f_{0} m_{j}\right)+f_{0} m_{j} \mathbf{c} \cdot \nabla_{y}\left(\beta_{i}^{j}\right)\right)=-\frac{f_{0}}{\rho_{0}} c_{i}
$$

Multiplying by $m_{k} / f_{0}$ and integrating in velocity space yields :

$$
\begin{aligned}
& \forall k \in\{1, N\} \quad \sum_{j=1}^{N}\left(-\frac{1}{f_{0}}\left\langle L\left(f_{0} m_{j}\right), m_{k}\right\rangle \beta_{i}^{j}+\left\langle m_{j} \mathbf{c}, m_{k}\right\rangle \cdot \nabla_{y}\left(\beta_{i}^{j}\right)\right)= \\
& \ldots-\left\langle\frac{c_{i} m_{k}}{\rho_{0}}, 1\right\rangle
\end{aligned}
$$

where $\langle\bullet, \bullet\rangle$ denotes scalar product in $L^{2}(\mathbf{c})$ with $f_{0}$ as a weighing function. One recognizes then the following symmetrical hyperbolic problem :

$$
S \vec{\beta}_{i}+\sum_{p=1}^{3} A_{p} \nabla_{y} \vec{\beta}_{i}=\vec{g}
$$

where :

$$
\begin{array}{r}
S_{k j}=\frac{1}{f_{0}}\left\langle L\left(f_{0} m_{j}\right), m_{k}\right\rangle \\
\left(A_{p}\right)_{k j}=\left\langle c_{p} m_{j}, m_{k}\right\rangle \\
g_{k}=\left\langle\frac{c_{i} m_{k}}{\rho_{0}}, 1\right\rangle
\end{array}
$$


In addition to this, boundary conditions may be written in the same formalism, representing the constant 1 function as $1=\sum_{j=1}^{N} \theta_{j} m_{j}$ :

$$
\left\{\begin{array}{l}
\sum_{p=1}^{3} \mathbf{n}_{p} A_{p} \vec{\beta}_{i} \cdot \vec{\theta}=0 \text { on } \partial Y_{f s} \\
\sum_{p=1}^{3} \mathbf{n}_{p} A_{p}^{+} \vec{\beta}_{i}=\sum_{p=1}^{3} \mathbf{n}_{p} A_{p}^{+} K_{i} \vec{\theta} \text { on } \partial Y_{f s}
\end{array}\right.
$$

where the $A$ matrix may be split into a positive and a negative contribution : $A=A^{+}+A^{-} . A^{+}$has the same eigenvector basis set as $A$ but its eigenvalues are the positive parts of the eigenvalues of $A$.

This implies that:

$$
\sum_{p=1}^{3} \mathbf{n}_{p}\left(A_{p}^{+}+A_{p}^{-}\right) \overrightarrow{\beta_{i}} \cdot \vec{\theta}=0 \text { on } \partial Y_{f s}
$$

The $K_{i}$ coefficients may be evaluated in the following way :

— The $\beta_{i}$ are also split into an impinging and an outgoing contribution :

$$
\beta_{i}^{o}=\sigma \theta+(1-\sigma) \beta_{i}^{i}(\mathbf{c}-2 \mathbf{c} \cdot \mathbf{n} \mathbf{n}) \text { on } \partial Y_{f s}
$$

Eq. (30) then becomes :

$$
\sum_{p=1}^{3}\left(\sigma K A_{p}^{+} \theta+(1-\sigma) A_{p}^{+} \beta_{i}^{i}(\mathbf{c}-2 \mathbf{c} \cdot \mathbf{n} \mathbf{n})+A_{p}^{-} \beta_{i}^{i}\right)=0 \text { on } \partial Y_{f s}
$$

— By symmetry of the specular collisions, it is noted that :

$$
A^{+} \beta(\mathbf{c}-2 \mathbf{c} \cdot \mathbf{n})=-A^{-} \beta
$$

Eq. (32) then becomes :

$$
\sum_{p=1}^{3} \sigma \mathbf{n}_{p}\left(K A_{p}^{+} \theta-A_{p}^{-} \beta_{i}^{i}\right)=0 \text { on } \partial Y_{f s}
$$

This gives finally an expression for $K$ :

$$
K_{i}=\frac{\sum_{p=1}^{3} \mathbf{n}_{p} A_{p}^{-} \beta_{i}^{i}}{\sum_{p=1}^{3} \mathbf{n}_{p} A_{p}^{+} \theta}
$$

Eqs. (25) and (29) are then much less computationally expensive than the original kinetic problem formulated in $\mathbb{R}^{6}$. However, the 
choice of a convenient basis set is a crucial issue for the quality of the results. Such a choice is conveniently guided by the analysis of the continuum and pure Knudsen regimes. In the continuum regime, remembering that the zero-order expansion of $\beta$ is described by (Vignoles et al., 2008) :

$$
\begin{aligned}
\alpha^{(-1)} & =b_{0}+\mathbf{b}_{1} \cdot \mathbf{c}+b_{2}|c|^{2} \\
\beta^{(0)} & =d_{0}+\mathbf{d}_{1} \cdot \mathbf{c}+d_{2}|c|^{2}
\end{aligned}
$$

and choosing an orthogonal set suited for the description of $\beta$ and $\mathbf{c} \beta$, the thirteen following moments are retained :

$$
\begin{gathered}
m_{1}=1 \\
m_{2}=\frac{c_{1}}{\sqrt{\frac{\mathcal{R} T_{0}}{M}}}, m_{5}=\frac{c_{1}^{2}-\frac{\mathcal{R} T_{0}}{M}}{\sqrt{2} \frac{\mathcal{R} T_{0}}{M}}, m_{8}=\frac{c_{1} c_{2}}{\frac{\mathcal{R} T_{0}}{M}}, m_{11}=\frac{|c|^{2}-5 \frac{\mathcal{R} T_{0}}{M}}{\sqrt{10\left(\frac{\mathcal{R} T_{0}}{M}\right)^{3}}} c_{1}, \\
m_{3}=\frac{c_{2}}{\sqrt{\frac{\mathcal{R} T_{0}}{M}}}, m_{6}=\frac{c_{2}^{2}-\frac{\mathcal{R} T_{0}}{M}}{\sqrt{2} \frac{\mathcal{R} T_{0}}{M}}, m_{9}=\frac{c_{1} c_{3}}{\frac{\mathcal{R} T_{0}}{M}}, m_{12}=\frac{|c|^{2}-5 \frac{\mathcal{R} T_{0}}{M}}{\sqrt{10\left(\frac{\mathcal{R} T_{0}}{M}\right)^{3}}} c_{2}, \\
m_{4}=\frac{c_{3}}{\sqrt{\frac{\mathcal{R} T_{0}}{M}}}, m_{7}=\frac{c_{3}^{2}-\frac{\mathcal{R} T_{0}}{M}}{\sqrt{2} \frac{\mathcal{R} T_{0}}{M}}, m_{10}=\frac{c_{2} c_{3}}{\frac{\mathcal{R} T_{0}}{M}}, m_{13}=\frac{|c|^{2}-5 \frac{\mathcal{R} T_{0}}{M}}{\sqrt{10\left(\frac{\mathcal{R} T_{0}}{M}\right)^{3}}} c_{3} .
\end{gathered}
$$

This basis is such that any solution belonging to the vector space generated by $\left(1, \mathbf{c},|c|^{2}\right)$ be exactly represented, as stated.

In the free-molecule limit, where the collision operator vanishes, the result of the Knudsen limit analysis (Vignoles et al., 2008) yields a dependance of $\beta$ to other functions, which are not polynomials of $\mathbf{c}$, but are based on the normal vector associated to $\mathbf{c}$, namely $n_{i}=\frac{c_{i}}{|c|}$. So, it is chosen to add the following moments to the preceding ones :

$$
m_{14-22}^{\prime}=\left(n_{1}, n_{2}, n_{3}, n_{1}^{2}, n_{2}^{2}, n_{3}^{2}, n_{1} n_{2}, n_{1} n_{3}, n_{2} n_{3}\right)
$$

However, it is recognized that the sum $n_{1}^{2}+n_{2}^{2}+n_{3}^{2}=1$, so one of the moments has to be excluded from the list. It is chosen to symmetrize the set by dropping $m_{1}$, so the complete basis set has 21 moments.

The discretization in space has been performed with a secondorder finite volume scheme. The system to be solved is linear, with 21 degrees of freedom per node, and a preconditioned GMRES algorithm is used for the resolution. Indeed, preconditioning improves 
CPU time (s)

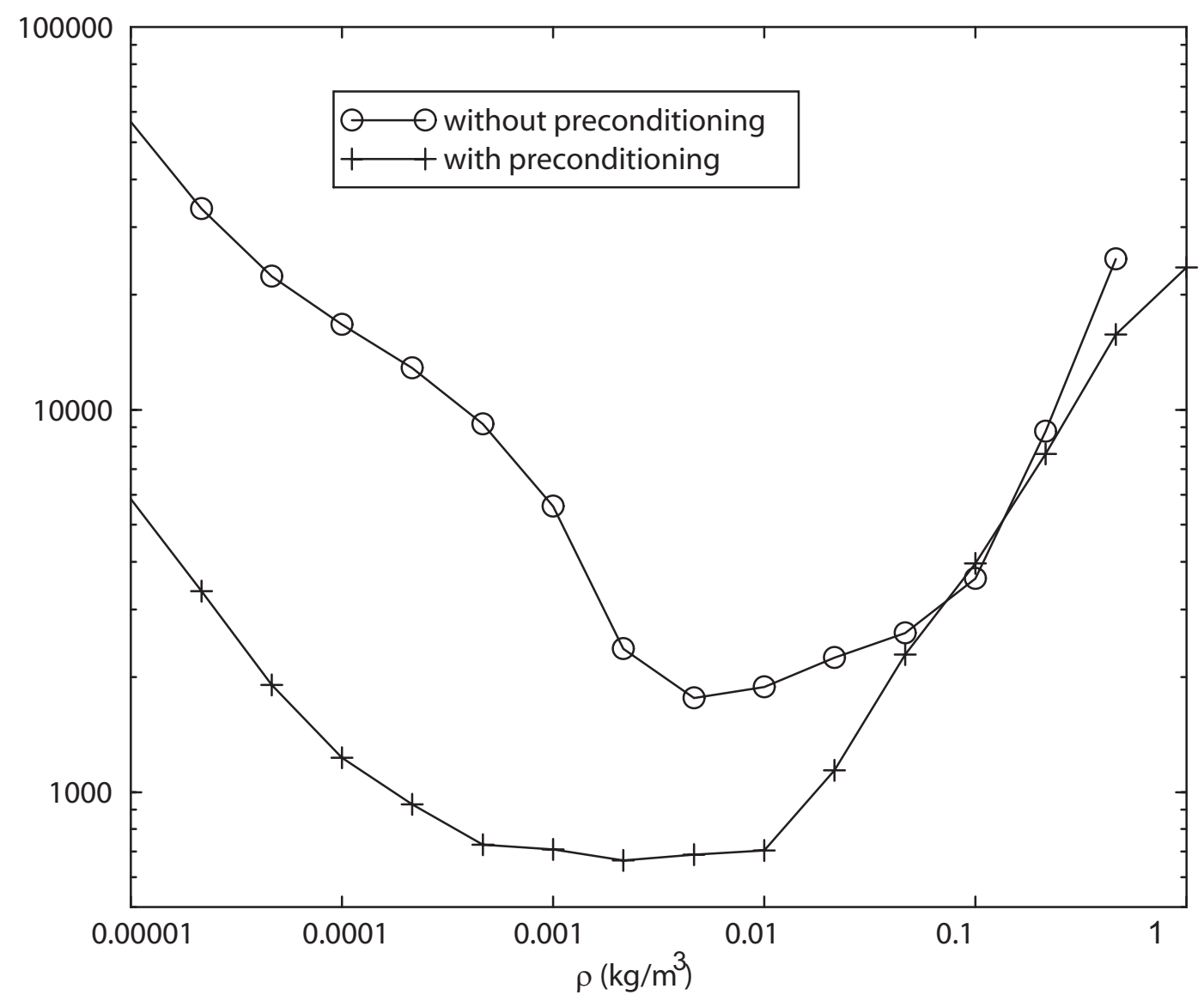

Figure 1. CPU time for the computation of $D$ vs. gas density with and without preconditioner.

substantially the convergence of the GMRES method. A good tradeoff between the efficiency and the overhead cost was obtained with the Symmetric Gauss-Seidel preconditioner. Figure 1 shows the efficiency of the preconditioning in terms of CPU time. For some regimes the CPU time is divided by a factor greater than 10 . We notice that the preconditioner is more efficient in transitional and rarefied regimes. The numerical difficulties are more important in the rarefied regime because of the computation of the coefficients $K_{i}$. Also, going to the continuum regime diminishes the efficiency of the preconditioner, because the nature of the equations to be solved becomes very different (i. e. they become fully elliptical), and the preconditioner has not been 
optimized to address this asymptotic limit for general geometries. Nonetheless, whatever the regime, the solver has proved to be able to solve large systems up to 1700000 unknowns, as those arising in the test case presented in section 4.3. Exterior boundary conditions are addressed as usual in finite-volume methods, that is, with fictitious nodes placed outside the resolution domain, and at which the value of the unknowns are given by mirroring relationships.

\subsubsection{Integration of the balance equations}

The macroscopic model that has to be solved is taken in its stationary form :

$$
\begin{aligned}
-\operatorname{div}_{x}\left(\tilde{D} \nabla_{x} T+D \nabla_{x} \rho\right) & =0 \\
-\operatorname{div}_{x}\left(\tilde{K} \nabla_{x} T\right) & =0 \\
T & =T_{1} \text { at } x=0 \\
T & =T_{2} \text { at } x=L \\
\rho & =\frac{M P_{2}}{\mathcal{R} T_{2}} \text { at } x=L \\
\tilde{D} \nabla_{x} T+D \nabla_{x} \rho & =0 \text { at } x=L
\end{aligned}
$$

Since heat transfer is guaranteed by the pore walls in the experimental configuration, and that the coupling is weak (i.e. the gas receives its temperature from the walls), it may be assumed that the gas temperature follows a linear gradient.

One has finally to solve the following ODE :

$$
\frac{\partial \rho}{\partial x}=\frac{\tilde{D}(\rho, T)}{D(\rho, T)} \frac{\partial T}{\partial x}
$$

subject to boundary condition :

$$
\rho(x=L)=\frac{P_{2}}{\mathcal{R} T_{2}}
$$

This is a straightforward task provided the dependencies of the coefficients $D$ and $\tilde{D}$ are given, e. $g$. through a tabulation.

\subsection{Results And Discussion}

The results will be discussed first with respect to other theoretical approaches yielding local effective coefficients in a single straight 
cylindrical pore. Then, a validation with respect to the presented experimental data will be given.

\subsubsection{Change-of-scale results}

Figure 2 illustrates the convergence of the results for $D$ with respect to the grid definition. It is seen that convergence is slower in the continuum regime than in the Knudsen regime. This was to be expected since the numerical procedure was specifically designed to capture the low-density behavior.

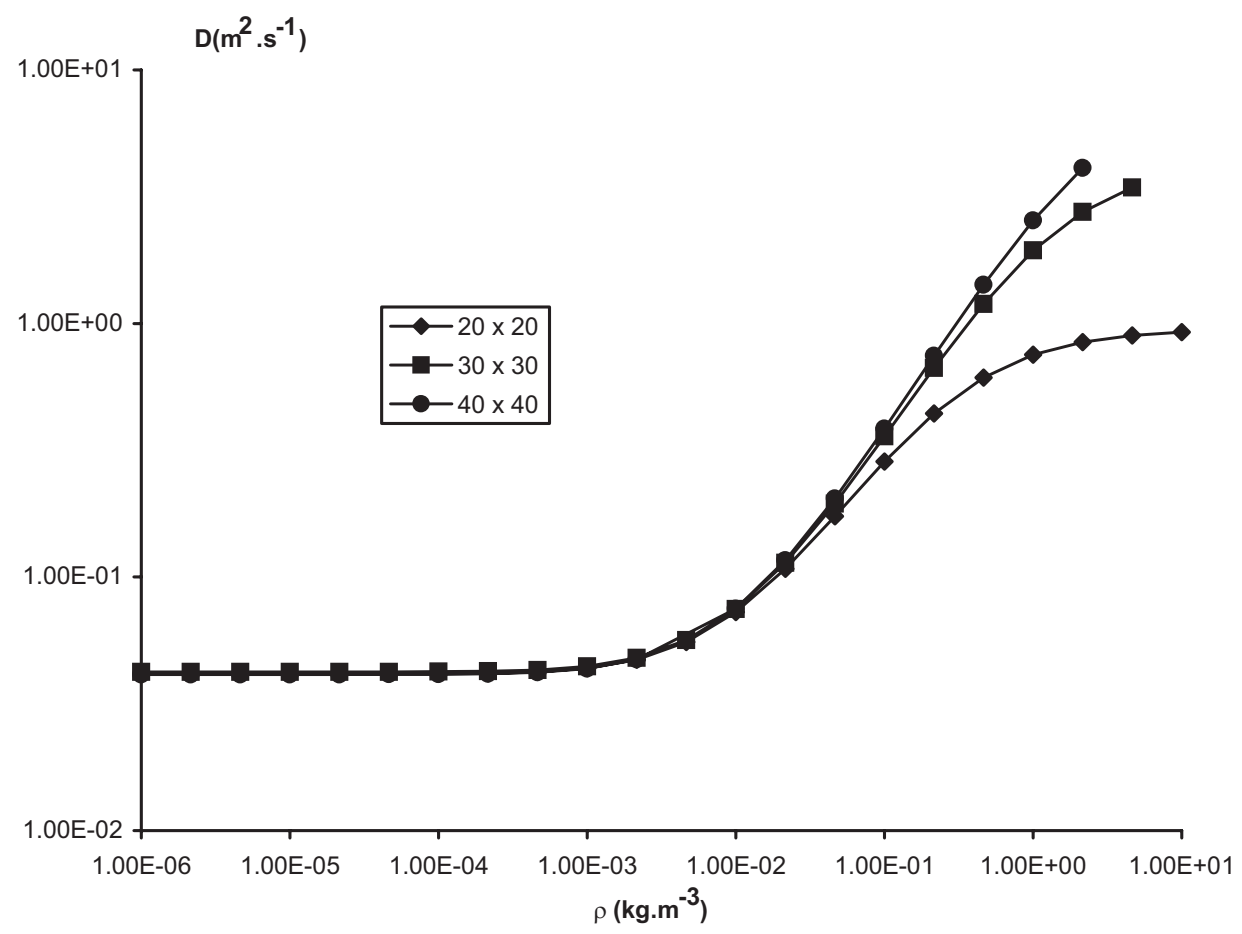

Figure 2. Coefficient $D$ as a function of gas density $\rho$ for various discretization parameters at $T=400 \mathrm{~K}$, for a cylindrical pore. The accommodation coefficient is $\sigma=0.6$ and the ES-BGK scheme has been used.

Figures 3 and 4 are plots of the evolution of the longitudinal $D$ and $\tilde{D}$ coefficients as a function of $\rho$ for argon at $400 \mathrm{~K}$, with a unit accommodation factor (i.e perfectly diffuse reflection at walls, $\sigma=1$ ). A comparison with the DGM formulae (here in scalar isotropic form):

$$
D_{K}=\frac{\epsilon}{3} \bar{c} d_{p} \cdot \frac{1}{1+\sigma \frac{\pi}{8}}
$$




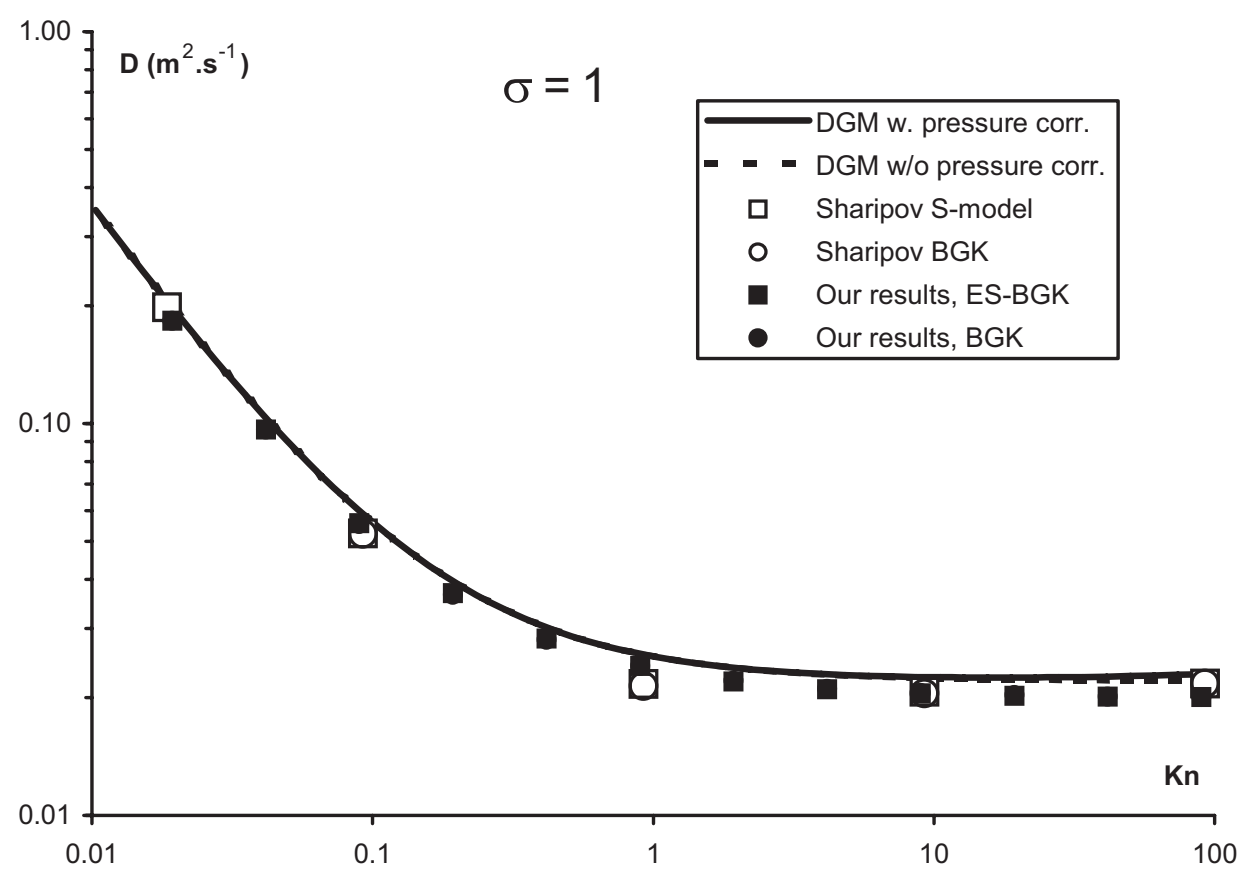

Figure 3. Coefficient $D$ as a function of Knudsen number $K n=\frac{\eta}{\rho D_{K, \text { ref }}}$ at $T=400 \mathrm{~K}$, for a cylindrical pore. The accommodation coefficient $\sigma$ is unity.

$$
\begin{aligned}
B & =\epsilon \frac{d_{p}^{2}}{32} \\
D & =D_{K}+\frac{P B}{\eta} \\
\tilde{D} & =\frac{\rho}{T}\left(\frac{P B}{\eta}+D_{K}\left(1+\alpha_{T}\right)\right)
\end{aligned}
$$

and with BGK and s-model (Sharipov, 1996) results with a unit accommodation coefficient show that all approaches are in good agreement with each other. It is to be noted that the computations performed in the present frame do not feature the small pressure dependency of the Knudsen diffusion coefficient, in contrast to Sharipov's computations.

From figure 5 it is seen that the ratio $\frac{\tilde{D} T}{D \rho}$ indeed varies from $1 / 2$ at high Knudsen numbers to 1 in the continuum limit. Some discrepancies between the approaches are visible in the intermediate regime : they are due to the fact that since plain BGK computations fail to reproduce the correct Prandtl number for the gas, they do not 


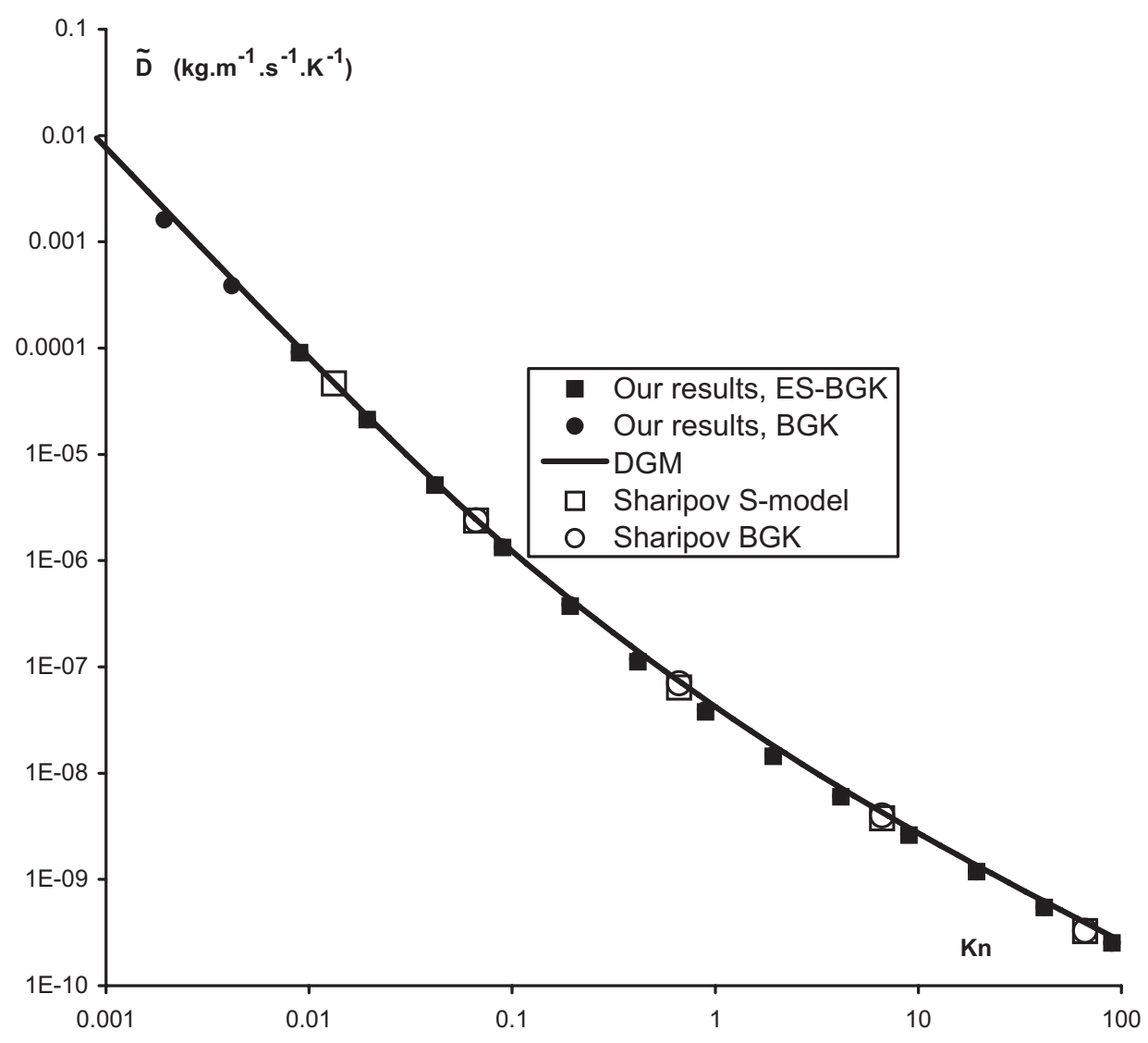

Figure 4. Coefficient $\tilde{D}$ as a function of Knudsen number $K n$ at $T=400 K$, for a cylindrical pore. The accommodation coefficient $\sigma$ is unity.

provide as good approximations as the ES-BGK ones. The DGM prediction seems to overestimate the ratio as compared to any numerical approach.

Finally, figure 6 is a plot of $D$ vs. $K n / \xi$ for various values of $\sigma$ (division of the abscissa by $\xi$ allows a superposition of the predictions in continuum regime). It is clear that the estimation of the DGM yields a lesser sensitivity of the Knudsen regime limit coefficient to $\sigma$ than actually computed by either method.

In summary, even some minute discrepancies with related results are present, the computational procedure for the change-of-scale may be considered as validated against well-known results. 


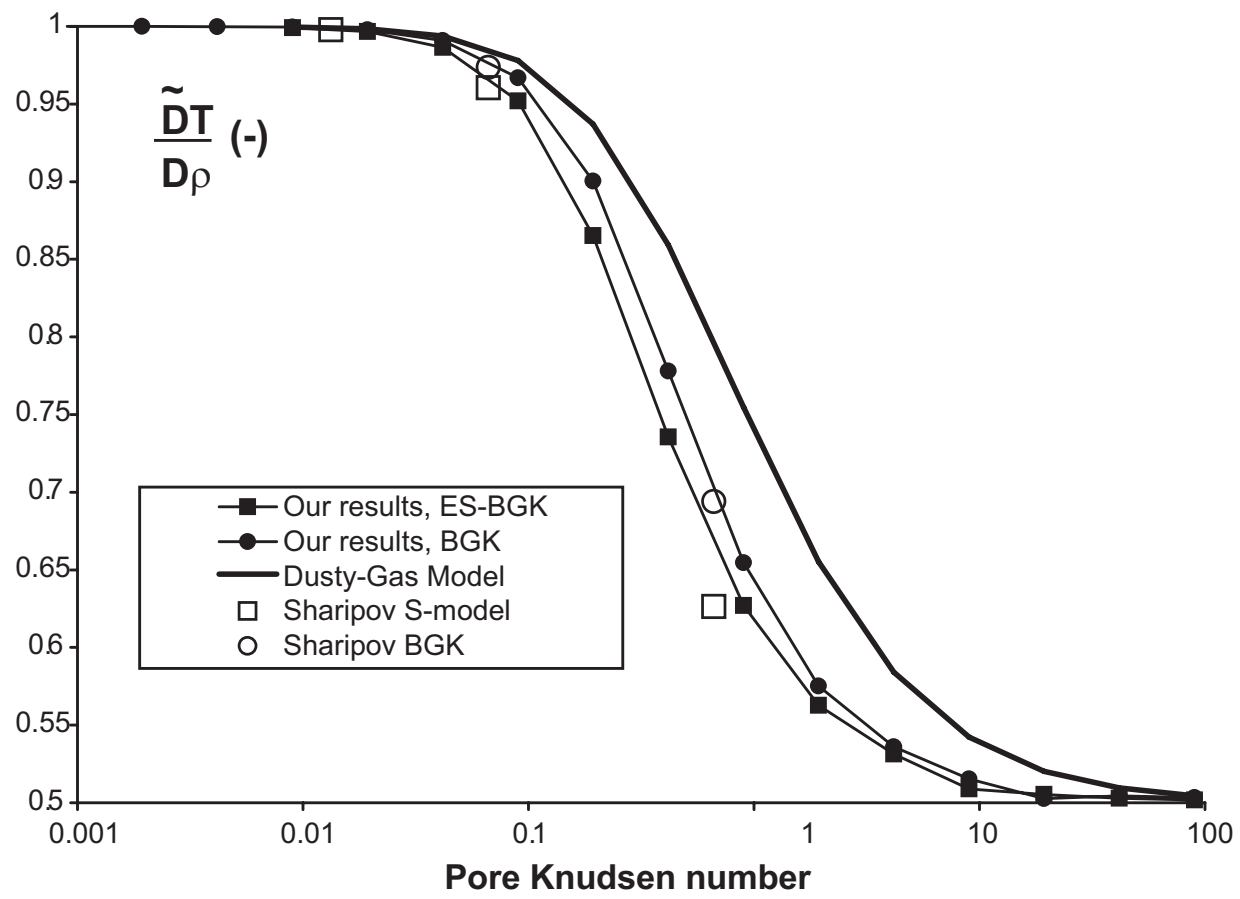

Figure 5. Coefficient ratio $\frac{\tilde{D} T}{D \rho}$ as a function of Knudsen number $K n$ at $T=400 K$ for a cylindrical pore. The accommodation coefficient $\sigma$ is unity.

\subsubsection{Validation with respect to experimental results}

Figure 7 is a plot of the pressure drop along the tube in the aforementioned experiment, together with predictions from our computational procedure. One parameter is not known a priori in the modelling procedure : the accommodation coefficient $\sigma$. It is usually claimed to be close to 1 for many gases and various walls; however, it has been chosen to make it vary, and a good fit is achieved for $\sigma=0.65$.

On performing these computations, it has been realized that the main parameter influencing the adequation of the model to the experimental data is the thermal transpiration ratio $\tilde{D} T / D \rho$. When the accommodation parameter $\sigma$ varies, the curves for $\tilde{D}(\rho)$ and $D(\rho)$ do not display a strong variation, while the transpiration ratio has a much more marked variation, which explains the found sensitivity. 


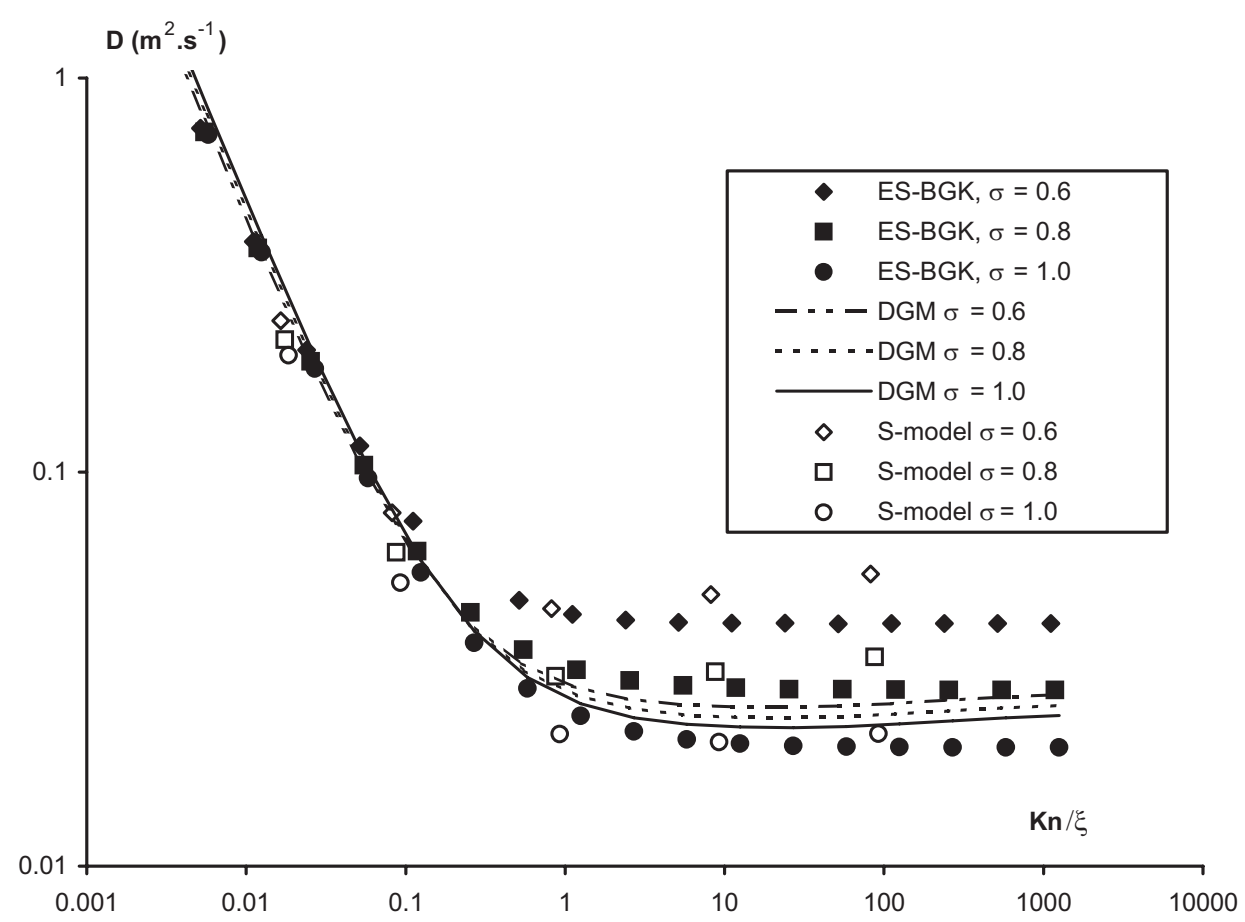

Figure 6. Coefficient $D$ as a function of scaled Knudsen number $K n / \xi$ at $T=400 \mathrm{~K}$ for a cylindrical pore, with various values of the accommodation coefficient $\sigma$.

\section{Results and discussions for various porous media}

Once the numerical procedure has been checked against results available for very simple geometries (a straight cylindrical tube), it may be used on more complicated porous media images, in order to assess the impact of the geometry on transport in continuum, rarefied, and intermediate regime. We will first present families of porous media images, and then discuss the results for transport as a function of the principal geometrical parameters.

\subsection{Test medium $1: 2 \mathrm{D}$ Anisotropy}

This ideal porous media 3D image contains 2 plates parallel to $z$, between which some obstacles have been located. Periodic boundary conditions have been given in $x$ and $y$ directions. Blocking the flux between parallel plates is a convenience to avoid any "infinite horizon" problem, for which it is well known that the Knudsen effective diffusivity diverges. The shape of the obstacles is such that the flow 


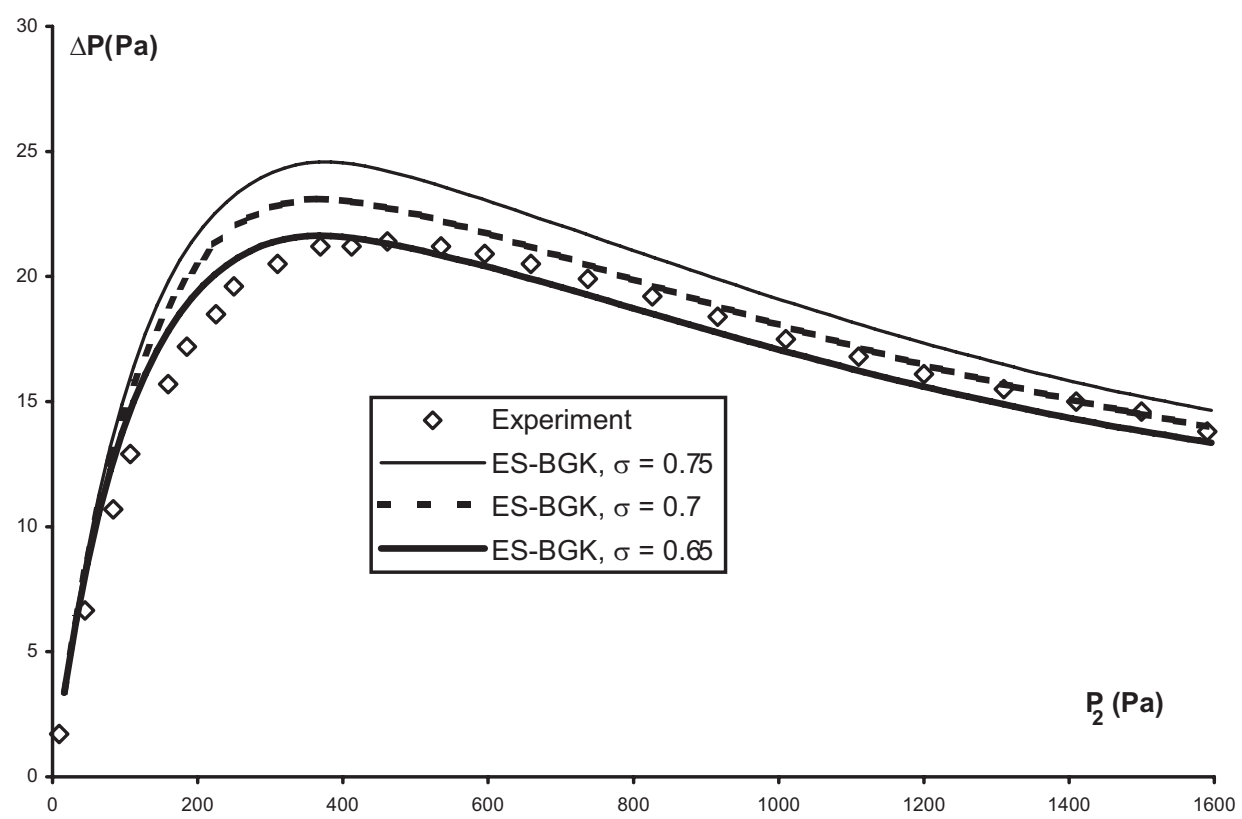

Figure 7. Computed vs. experimental curves of pressure drop in thermal transpiration experiment.

field cannot display any symmetry axis. The principal directions for the effective property tensors are thus not obvious a priori from a simple glance to an $(x, y)$ section of the $3 \mathrm{D}$ images. Figure 8 is a visualization of this medium.
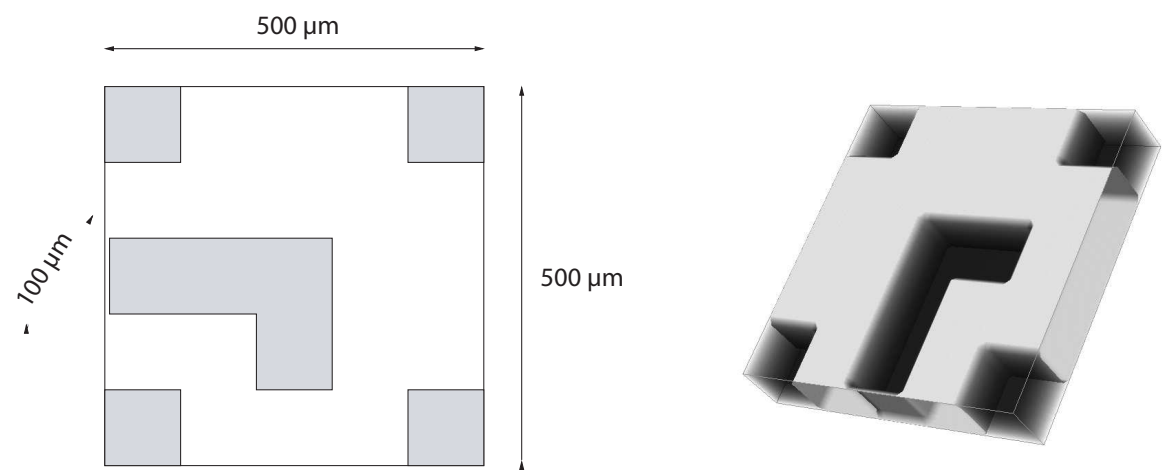

Figure 8. Visualization of the test porous medium unit cell $n^{\circ} 1$.

The results on tortuosity factors are summarized at figure 9, and compared with prediction by two other codes : a Monte-Carlo Ran- 


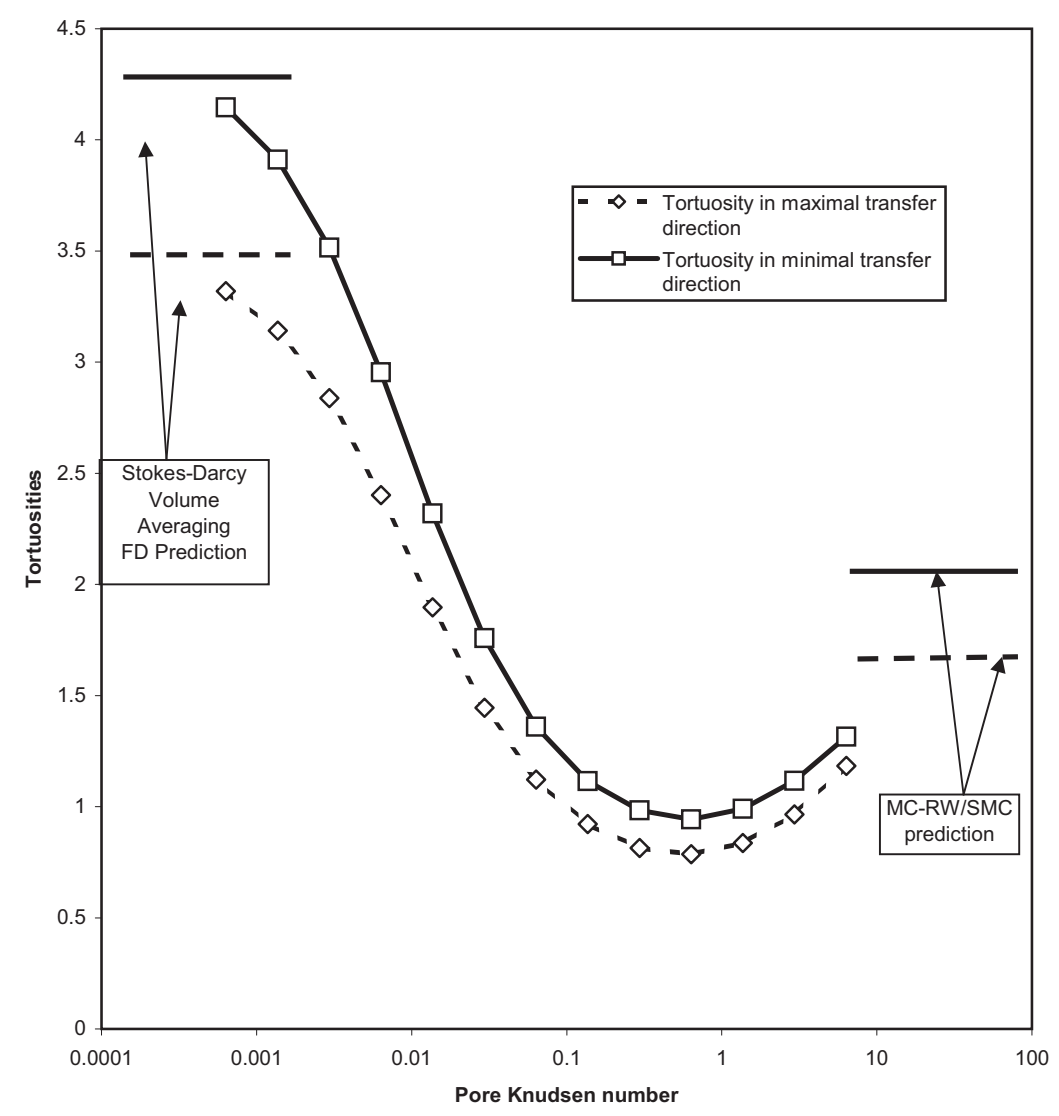

Figure 9. Tortuosity evaluation of test porous medium $\mathrm{n}^{\circ} 1$ with respect to mass transfer : comparison of the presented code and of two independent estimations of the continuum limit (Volume Averaging FD) and of the rarefied limit (MC-RW/SMC)

dom Walks code with Simplified Marching Cube discretization of the fluid-solid interface (Vignoles, 1995) for the rarefied gas limit, and a numerical solver for the continuum regime Stokes-to-Darcy problem resolution, based on the volume averaging theory (Anguy et al., 1994). The agreement is excellent on the continuum limit, and of somewhat lower quality, though satisfactory, on the rarefied limit : this indicates that there is some difficulty for the code to capture the extremely rarefied gas behavior. Note that the tortuosity factors display a minimal value for $K n$ numbers around unity : this means that when both transport modalities are of comparable magnitude, they are able to combine themselves in order to better transfer gas through the medium. Consequently, describing transport with a direct 


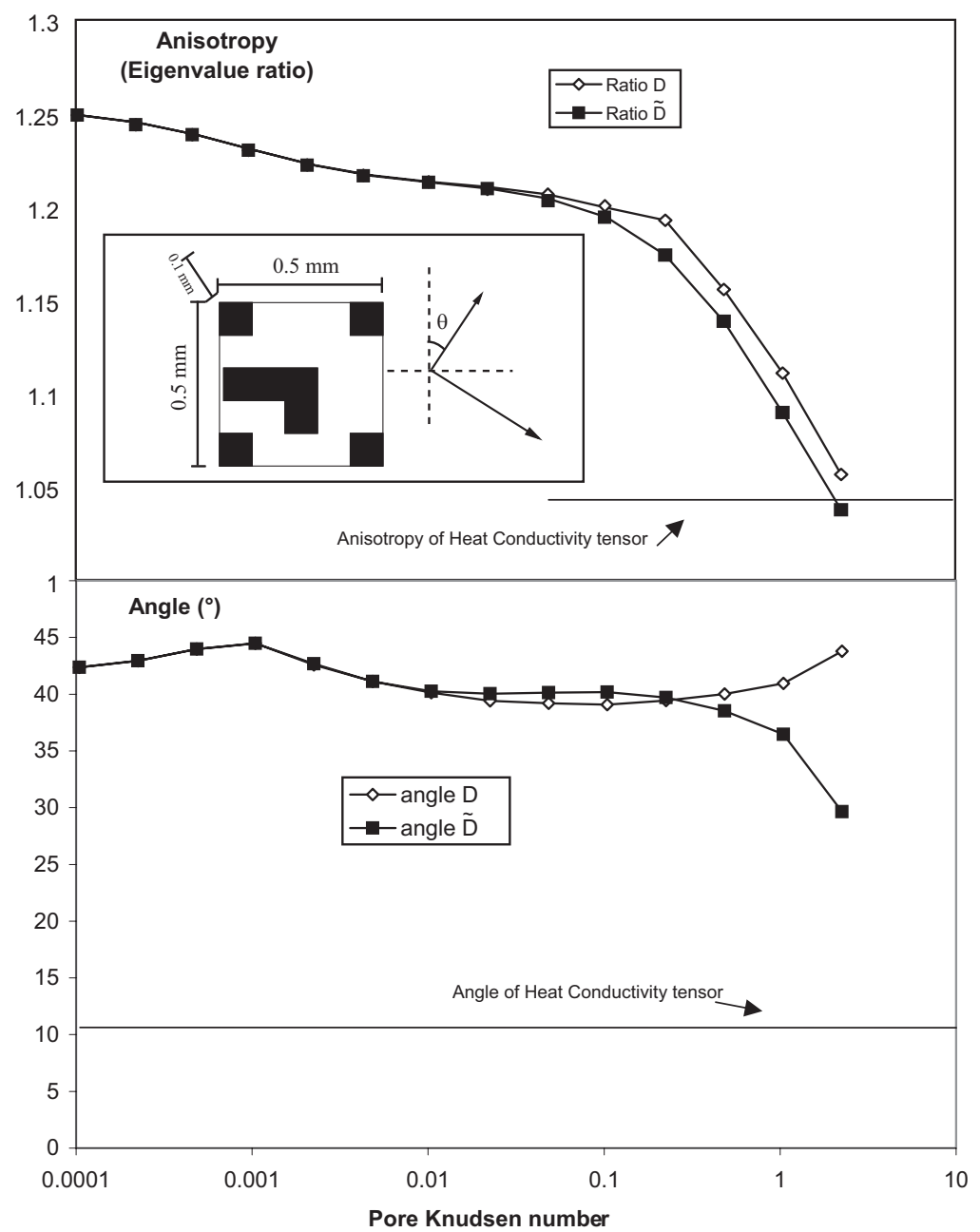

Figure 10. Angles and anisotropy ratios of $D$ and $\tilde{D}$ principal tensor components for porous medium $\mathrm{n}^{\circ} 1$ as a function of the pore Knudsen number. The values for the heat conductivity tensor are also provided for comparison.

interpolation between the two asymptotic behaviors would result in a wrong description of the involved physics.

The evolution of the anisotropy ratio and the orientations of the two tensors $\underline{\underline{D}}$ and $\underline{\underline{D}}$ are reported at figure 10 . It is seen clearly that the anisotropy of both tensors diminishes when the regime becomes rarefied; this diminution is slightly more marked for $\underline{\underline{D}}$, a fact that may be related to the neatly lower anisotropy of the heat conduction tensor. The orientation of the principal axes does not change apprecia- 
bly, except for $\underline{\underline{D}}$ at high Knudsen numbers : this can be interpreted as a realignment with respect to the heat transfer tensor. This confirms that the $\alpha_{T}$ parameter of the Dusty-Gas Model, should be rewritten in a tensorial form.

\subsection{Test image $2: 3 \mathrm{D}$ anisotropy}

After having investigated the possible separation of the angular directions of the tensors $\underline{\underline{D}}$ and $\underline{\underline{D}}$ when Knudsen number incresases, it has been tried to generate a porous medium for which the anisotropy ratios can differ significantly for these two transfer modalities. The test medium 2 is the periodic $3 \mathrm{D}$ repetition of the unit cell sketched at figure 11. It consists in a square tube sectioned by a non-planar "crack", or void slice. The non-planarity of the crack has been chosen so that the condition of finite horizon be respected, in order to get a true Knudsen diffusion tensor at the rarefied limit. The tube volume and the slice volume are equal.

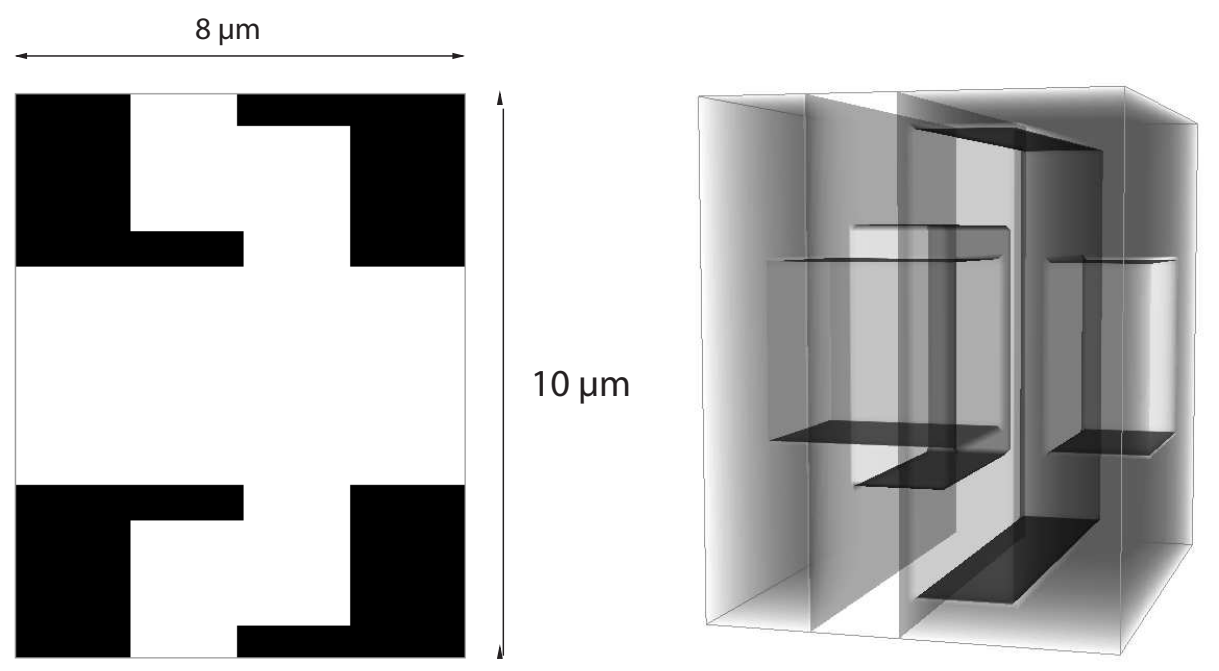

Figure 11. Visualization of the test porous medium unit cell $\mathrm{n}^{\circ} 2$.

Figure 12 is a plot of the anisotropy ratio as a function of gas density in the medium : the interesting fact is that while the direction of highest pressure-induced mass transportability $(\underline{\underline{D}})$ is always on the same side, the direction of highest thermal-induced mass transportability $(\underline{\underline{\tilde{D}}})$ is reversed when density decreases. This illustrates again the geometrical independence of Knudsen slip and thermal creep in a general porous medium. 


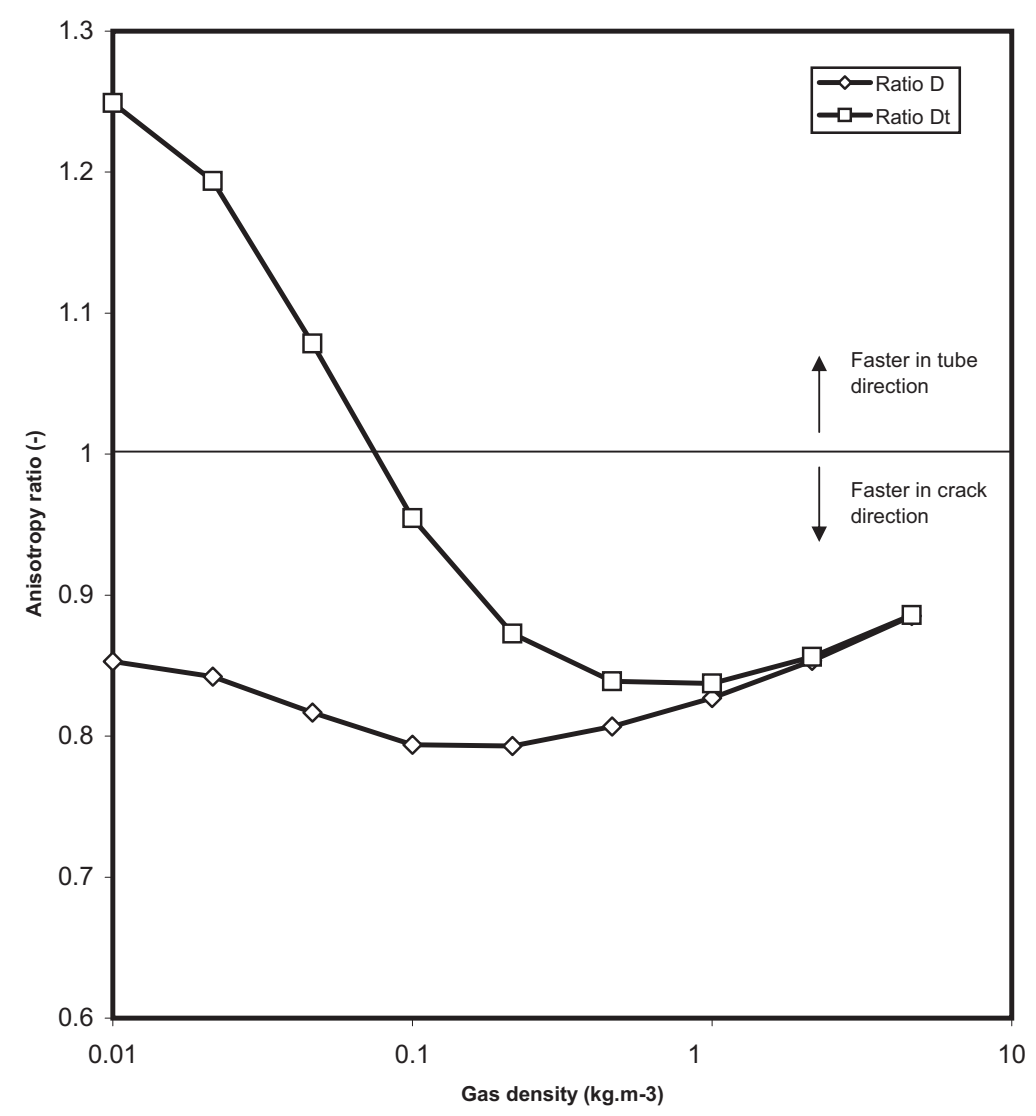

Figure 12. Anisotropy ratios of $D$ and $\tilde{D}$ principal tensor components for porous medium $\mathrm{n}^{\circ} 2$ as a function of gas density.

\subsection{Test medium 3 : Complex medium ACQuired By Synchrotron X-ray Computerized Tomography (SXCT)}

Finally, the developed numerical tools have been tested on a more realistic medium. Carbon fiber cloth stackings, which constitute preforms for $C / C$ composite materials, have been scanned in synchrotron $\mathrm{X}$ ray Computerized Microtomography (Coindreau and Vignoles, 2004) with $0.7 \mu \mathrm{m}$ resolution, resulting in excellent 3D images, thanks to an adequate segmentation procedure (Vignoles, 2001). These highresolution images have been utilized for the estimation of geometrical properties (Coindreau and Vignoles, 2005), as well as tortuosities with respect to binary diffusion and Knudsen diffusion (Coindreau et al., 2005; Vignoles et al., 2007), on a large number of sub-images. Here, 
one sub-image has been extracted ; the resolution has been lowered by a factor two in order to manage acceptable computational times, and the image has been made periodic in $3 \mathrm{D}$.
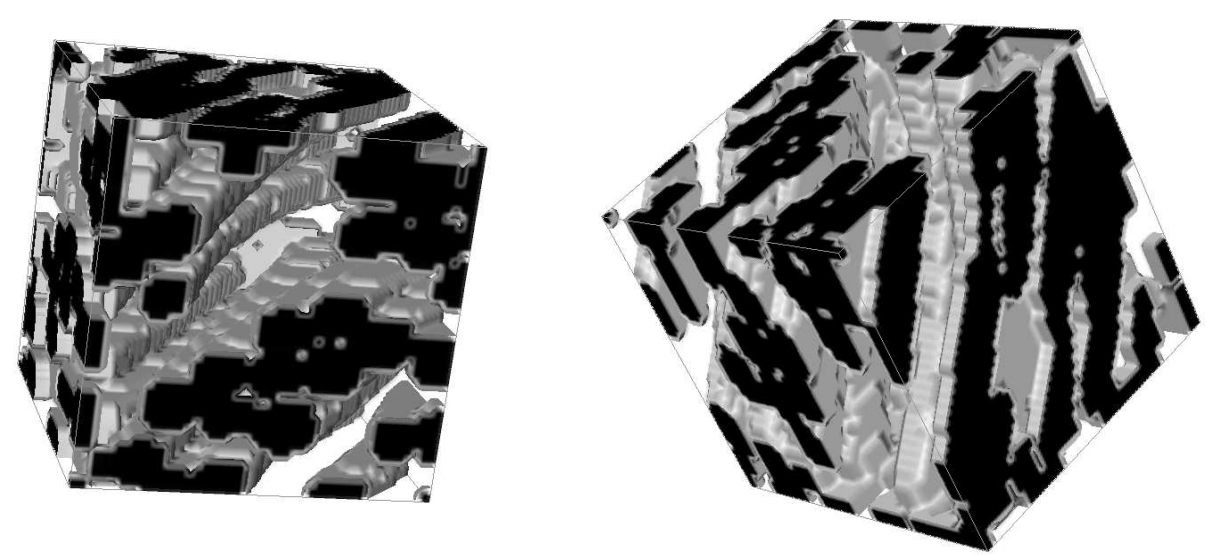

Figure 13. Visualizations of the test porous medium unit cell $\mathrm{n}^{\circ} 3$.
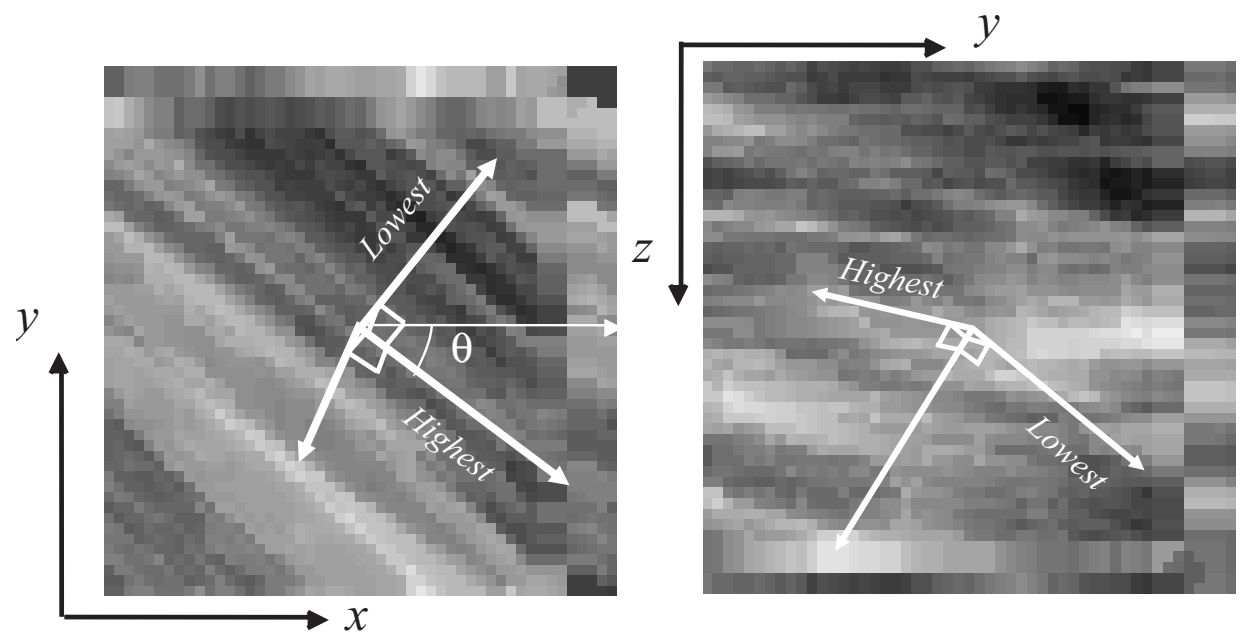

Figure 14. Visualizations of the principal tensor components for test porous medium unit cell $\mathrm{n}^{\circ} 3$ on $z$ and $x$ projections.

Figure 13 is a visualization of this test medium. The fibers are more or less parallel to the diagonal of one face of the cube. Figure 14 is a visualization of the tensor principal axes on $z$ and $x$ projections of this 3D image : it is seen that these directions indeed closely match the structure of the image. As could be expected, the direction of highest conductivity lies parallel to the fibers. The evolution of the anisotropy 


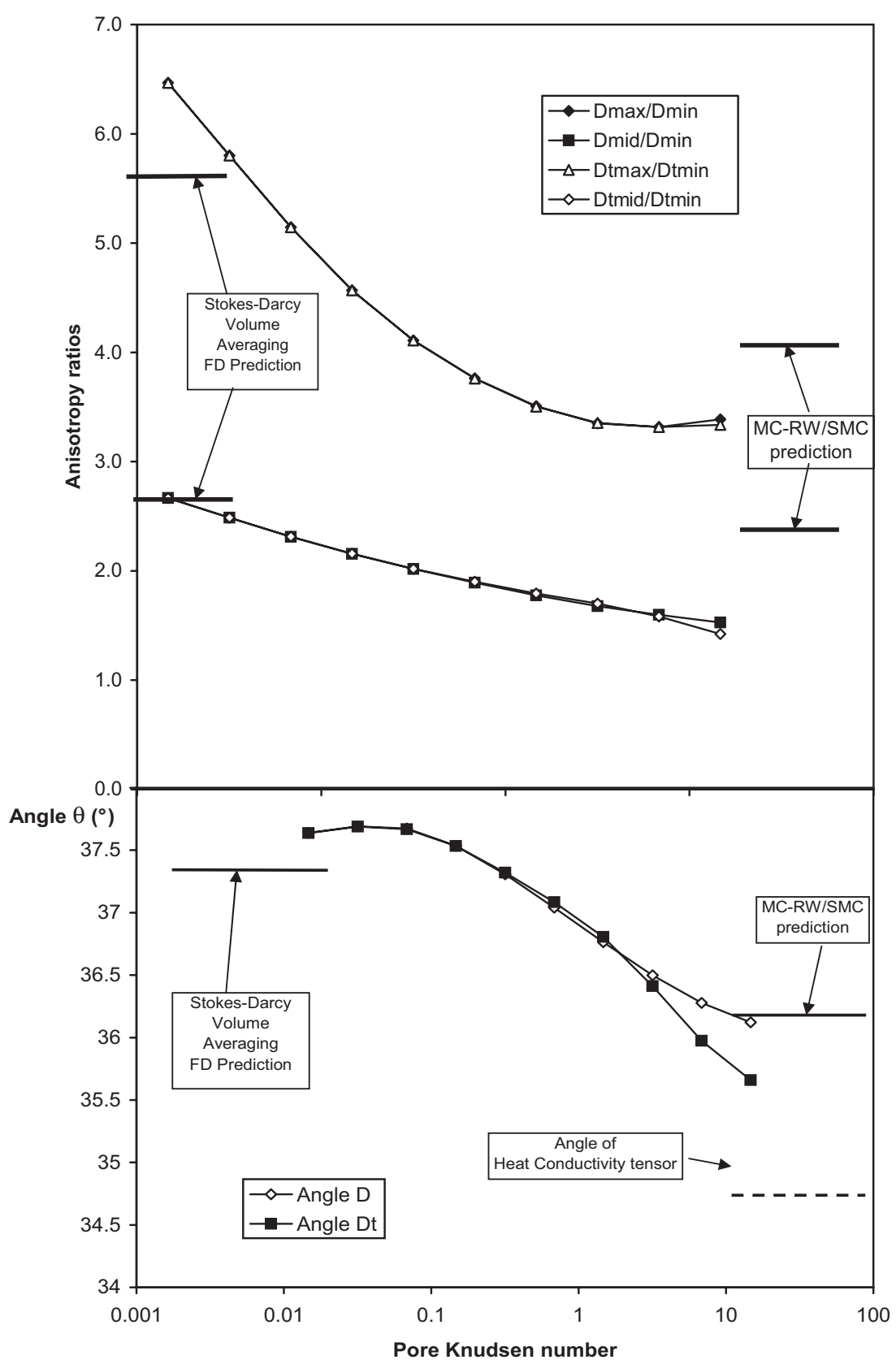

Figure 15. Anisotropy ratios of $D$ and $\tilde{D}$ principal tensor components for porous medium $\mathrm{n}^{\circ} 3$ as a function of gas density. The definition of angle $\theta$ refers to fig. 14 . 
ratio and of the orientation of tensors $\underline{\underline{D}}$ and $\underline{\underline{D}}$ are reported at figure 15 and compared with the two others codes, as for the test medium 1 : again, the conclusions are similar, showing that the quality of the presented approach is equivalent for complex media as for simpler ones.

\section{Conclusion}

The non-isothermal transport of a pure rarefied gas in a porous medium has been treated with a change-of-scale procedure starting from the kinetic-theory level of description, and making use of the homogenization technique. Based on a homogenization model presented in a companion paper (Vignoles et al., 2008), numerical schemes have been produced for the computation of effective transport coefficients (i.e. Knudsen transport, viscous transport, and thermal transpiration coefficient) in whatever 3D image of a porous medium. They have been tested favorably against numerical results from other independent works focused on single-pore transport and against the straight-pore experimental data of (Malinauskas et al., 1970).

Finally, in order to demonstrate the generality of the developed procedure, some more complex porous media images have been tested. The variation of the tensorial components for each kind of coefficient has been studied, showing that the geometry effect on permeability on one hand and on thermal transpiration as well as Knudsen transport on the other hand is markedly different. Such a difference has been quantified in terms of angles and anisotropy ratios, and demonstrate that a thermal transpiration tensor may differ from a Knudsen diffusion tensor.

\section{Acknowledgements}

The authors wish to thank CEA (Commissariat à l'Energie Atomique) for support through a PhD grant to C. P. and G. Duffa for fruitful discussions. 


\section{References}

Andries, P., P. L. Tallec, J.-P. Perlat, and B. Perthame: 2000, 'The Gaussian-BGK model of Boltzmann equation with small Prandtl number'. Eur. J. Mech. B : Fluids 19(6), 813-830.

Anguy, Y., D. Bernard, and R. Ehrlich: 1994, 'The local change of scale method for modelling fluid flow in natural porous media I : Numerical tools'. Adv. Water Res. 17, 337-351.

Bardos, C., L. Dumas, and F. Golse: 1997, 'Diffusion approximation for billiards with totally accommodating scatterers'. J. Statis. Phys. 86, 351-375.

Bensoussan, A., J.-L. Lions, and G. C. Papanicolau: 1978, Asymptotic analysis for periodic structures, No. 5 in Studies in Mathematics and its Applications. Amsterdam: North-Holland.

Besmann, T. M., B. W. Sheldon, R. A. Lowden, and D. P. Stinton: 1991, 'Vapor-phase fabrication and properties of continuous-filament ceramic composites'. Science 253, 1104-1109.

Bhatnagar, P. L., E. P. Gross, and M. Krook: 1954, 'A model for collision processes in gases'. Phys. Rev. 94(3), 511-525.

Burganos, V. N. and S. V. Sotirchos: 1989, 'Knudsen diffusion in parallel, multidimensional, or randomly oriented pore structures'. Chem. Eng. Sci. 44, 2451.

Charrier, P. and B. Dubroca: 2003, 'Asymptotic transport models for heat and mass transfer in reactive porous media'. SIAM J. Multiscale Model. Simul. 2(1), 124-157.

Coindreau, O. and G. L. Vignoles: 2004, 'Computing Structural and Transport Properties of $C / C$ Composites from $3 D$ Tomographic Images'. In: R. Martins, C. Dias, E. Fortunato, I. Ferreira, H. Godinho, and R. Monteiro (eds.): Advanced Materials Forum II, Vol. 455-456 of Mater. Sci. Forum. Zurich, pp. 751-754, Trans. Tech. Publications.

Coindreau, O. and G. L. Vignoles: 2005, 'Assessment of geometrical and transport properties of a fibrous $C / C$ composite preform as digitized by X-ray computed microtomography. Part I : Image acquisition and geometrical properties'. J. Mater. Res. 20, 2328-2339.

Coindreau, O., G. L. Vignoles, and J.-M. Goyhénèche: 2005, 'Multiscale X-ray CMT of $C / C$ composites : a tool for properties assessment'. In: N. P. Bansal, J. P. Singh, and W. M. Kriven (eds.): Advances in Ceramic-Matrix Composites XI, Vol. 175 of Ceram. Trans. Westerville, OH, pp. 77-84, The American Ceramic Society.

Gupta, A. D. and T. S. Storvick: 1970, 'Analysis of the heat conductivity data for polar and nonpolar gases using thermal transpiration measurements'. J. Chem. Phys. $\mathbf{5 2}(2), 742-749$.

Holway, L. H.: 1966, 'Kinetic theory of shock structure using an ellipsoidal distribution function'. In: J. H. D. Leeuw (ed.): Rarefied Gas Dynamics I, Vol. 3 of Advances in Applied Mechanics Supplement. New York, pp. 193-215, Academic Press.

Loyalka, S. K.: 1969, 'Thermal transpiration in a cylindrical tube'. Phys. Fluids 12, 2301.

Malinauskas, A. P., J. W. G. Jr, and B. K. Annis: 1970, 'Rotational collision numbers of $\mathrm{N}_{2}, \mathrm{O}_{2}, \mathrm{CO}$, and $\mathrm{CO}_{2}$ from thermal transpiration measurements'. J. Chem. Phys. 53(4), 1317-1324.

Naslain, R. (ed.): 1985, Introduction aux matériaux composites, Vol. 2. Bordeaux: IMC/Editions du CNRS.

Naslain, R. and F. Langlais: 1990, 'Fundamental and practical aspects of the chemical vapor infiltration of porous substrates'. High Temperature Science 27, 221-235. 
Ofori, J. Y. and S. V. Sotirchos: 1996a, 'Multidimensional modelling of chemical vapor infiltration : Application to isobaric CVI'. Ceram. Inter. 23, 119-126.

Ofori, J. Y. and S. V. Sotirchos: 1996b, 'Structural model effects on the predictions of CVI models'. J. Electrochem. Soc. 143(6), 1962-1973.

Reuge, N. and G. L. Vignoles: 2005, 'Global Modelling of I-CVI : Effects of reactor control parameters on a densification'. J. Mater. Process. Technol. 166, 15-29.

Sharipov, F.: 1996, 'Rarefied gas flow through a long tube at any temperature ratio'. J. Vac. Sci. Technol. A 14(4), 2627-2635.

Skjetne, E. and J.-L. Auriault: 1999, 'New insights on steady, nonlinear flow in porous media'. Eur. J. Mech. B : Fluids 18(1), 131-145.

Starr, T. L. and A. W. Smith: 1990, '3D Modeling of forced flow thermal gradient CVI for ceramic composite fabrication'. In: T. M. Besmann and B. M. Gallois (eds.): Chemical Vapor Deposition of Refractory Metals and Ceramics, Vol. 168 of Mat. Res. Soc. Symp. Proc. Pittsburgh, Pennsylvania, pp. 55-60, Materials Research Society.

Storvick, T. S., H. S. Park, and S. K. Loyalka: 1978, 'Thermal transpiration : A comparison of experiment and theory'. J. Vac. Sci. Technol. A 15(6), 1844-1852.

Strieder, W. C.: 1971, 'Gaseous self-diffusion through a porous medium'. J. Chem. Phys. 54(9), 4050-4053.

Strieder, W. C. and S. Prager: 1968, 'Knudsen flow through a porous medium'. Phys. Fluids 11(12), 2544-2548.

Tomadakis, M. M. and S. V. Sotirchos: 1991, 'Effective Knudsen diffusivities in structures of randomly overlapping fibers'. AIChE J. 37, 74-86.

Vignoles, G. L.: 1995, 'Modelling binary, Knudsen, and transition regime diffusion inside complex porous media'. J. de Physique IV C5, 159-166.

Vignoles, G. L.: 2001, 'Image segmentation for hard X-ray phase contrast images of $C / C$ composites'. Carbon 39, 167-173.

Vignoles, G. L.: 2006, 'Modelling of CVI Processes'. Adv. Sci. Technol. 50, 97-106.

Vignoles, G. L., P. Charrier, C. Preux, and B. Dubroca: 2008, 'Rarefied pure gas transport in non-isothermal porous media : Effective transport properties from homogenization of the kinetic equation'. Transp. in Porous Media 73, 211-232 DOI:10.1007/s11242-007-9167-7.

Vignoles, G. L., O. Coindreau, A. Ahmadi, and D. Bernard: 2007, 'Assessment of geometrical and transport properties of a fibrous $C / C$ composite preform as digitized by X-ray computed micro-tomography. Part II : Heat and gas transport'. J. Mater. Res. 22(6), 1537-1550.

Vignoles, G. L., J.-M. Goyhénèche, P. Sébastian, J.-R. Puiggali, J.-F. Lines, J. Lachaud, P. Delhaès, and M. Trinquecoste: 2006, 'The film-boiling densification process for $C C$ composite fabrication : From local scale to overall optimization'. Chem. Eng. Sci. 61, 5336-5353.

Vignoles, G. L., N. Nadeau, C.-M. Brauner, J.-F. Lines, and J.-R. Puiggali: 2005, 'The Notion of Densification Front in CVI with Thermal Gradients'. In: E. Lara-Curzio, D. Zhu, and W. M. Kriven (eds.): Mechanical Properties and Performance of Engineering Ceramics and Composites : Proc. 29 $9^{\text {th }}$ Intl. Conf. on Advanced Ceramics and Composites, Vol. 26 of Ceram. Eng. and Sci. Proc. Westerville, OH, pp. 187-195, The American Ceramic Society.

Whitaker, S.: 1987. In: S. Whitaker and A. Cassano (eds.): Chemical Reactor Analysis : Concept and Design, Vol. 3 of Chemical Engineering Concepts and Reviews. New York: Taylor \& Francis, Chapt. 1, pp. 1-94. 


\section{List of figures}

- Figure 1. CPU time for the computation of $D$ vs. gas density with and without preconditioner.

- Figure 2. Coefficient $D$ as a function of gas density $\rho$ for various discretization parameters at $T=400 \mathrm{~K}$, for a cylindrical pore. The accommodation coefficient is $\sigma=0.6$ and the ES-BGK scheme has been used.

- Figure 3. Coefficient $D$ as a function of Knudsen number $K n=$ $\frac{\eta}{\rho D_{K, r e f}}$ at $T=400 \mathrm{~K}$, for a cylindrical pore. The accommodation coefficient $\sigma$ is unity.

- Figure 4. Coefficient $\tilde{D}$ as a function of Knudsen number $K n$ at $T=400 K$, for a cylindrical pore. The accommodation coefficient $\sigma$ is unity.

- Figure 5. Coefficient ratio $\frac{\tilde{D} T}{D \rho}$ as a function of Knudsen number $K n$ at $T=400 K$ for a cylindrical pore. The accommodation coefficient $\sigma$ is unity.

- Figure 6. Coefficient $D$ as a function of scaled Knudsen number $K n / \xi$ at $T=400 K$ for a cylindrical pore, with various values of the accommodation coefficient $\sigma$.

- Figure 7. Computed vs. experimental curves of pressure drop in thermal transpiration experiment.

- Figure 8. Visualization of the test porous medium unit cell $\mathrm{n}^{\circ} 1$.

- Figure 9. Tortuosity evaluation of test porous medium $\mathrm{n}^{\circ} 1$ with respect to mass transfer : comparison of the presented code and of two independent estimations of the continuum limit (Anguy et al., 1994) (Volume Averaging FD) and of the rarefied limit (MC-RW/SMC) (Vignoles, 1995).

- Figure 10. Angles and anisotropy ratios of $D$ and $\tilde{D}$ principal tensor components for porous medium $\mathrm{n}^{\circ} 1$ as a function of the pore Knudsen number. The values for the heat conductivity tensor are also provided for comparison.

- Figure 11. Visualization of the test porous medium unit cell $n^{\circ} 2$. 
- Figure 12. Anisotropy ratios of $D$ and $\tilde{D}$ principal tensor components for porous medium $\mathrm{n}^{\circ} 2$ as a function of gas density.

- Figure 13. Visualizations of the test porous medium unit cell $n^{\circ} 3$.

- Figure 14. Visualizations of the principal tensor components for test porous medium unit cell $n^{\circ} 3$ on $z$ and $x$ projections.

- Figure 15. Anisotropy ratios of $D$ and $\tilde{D}$ principal tensor components for porous medium $\mathrm{n}^{\circ} 3$ as a function of gas density. The definition of angle $\theta$ refers to fig. 14 . 
Figures

CPU time (s)

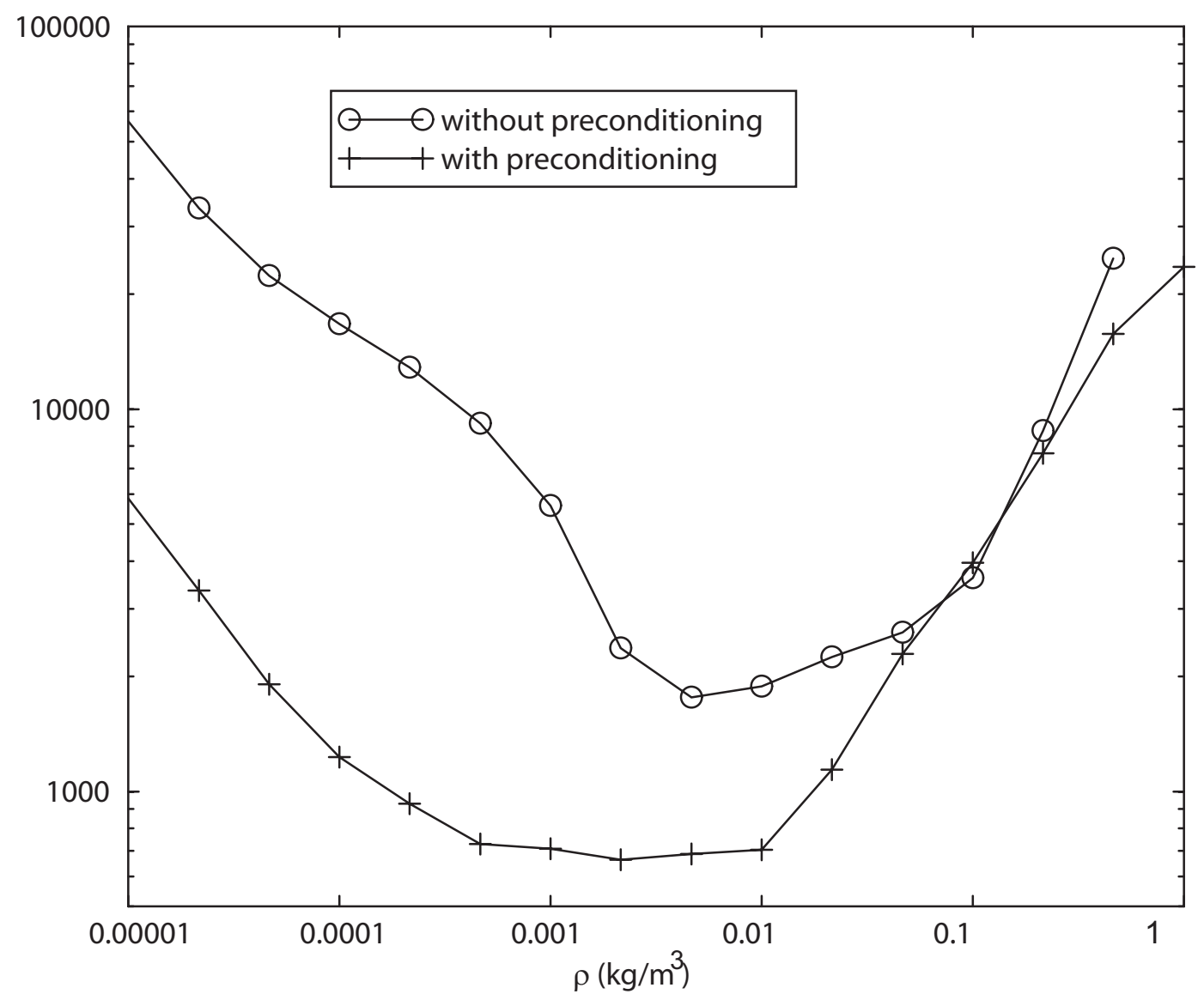

Figure 16. Preconditioner efficiency measured as CPU time for various values of pore Knudsen number. 


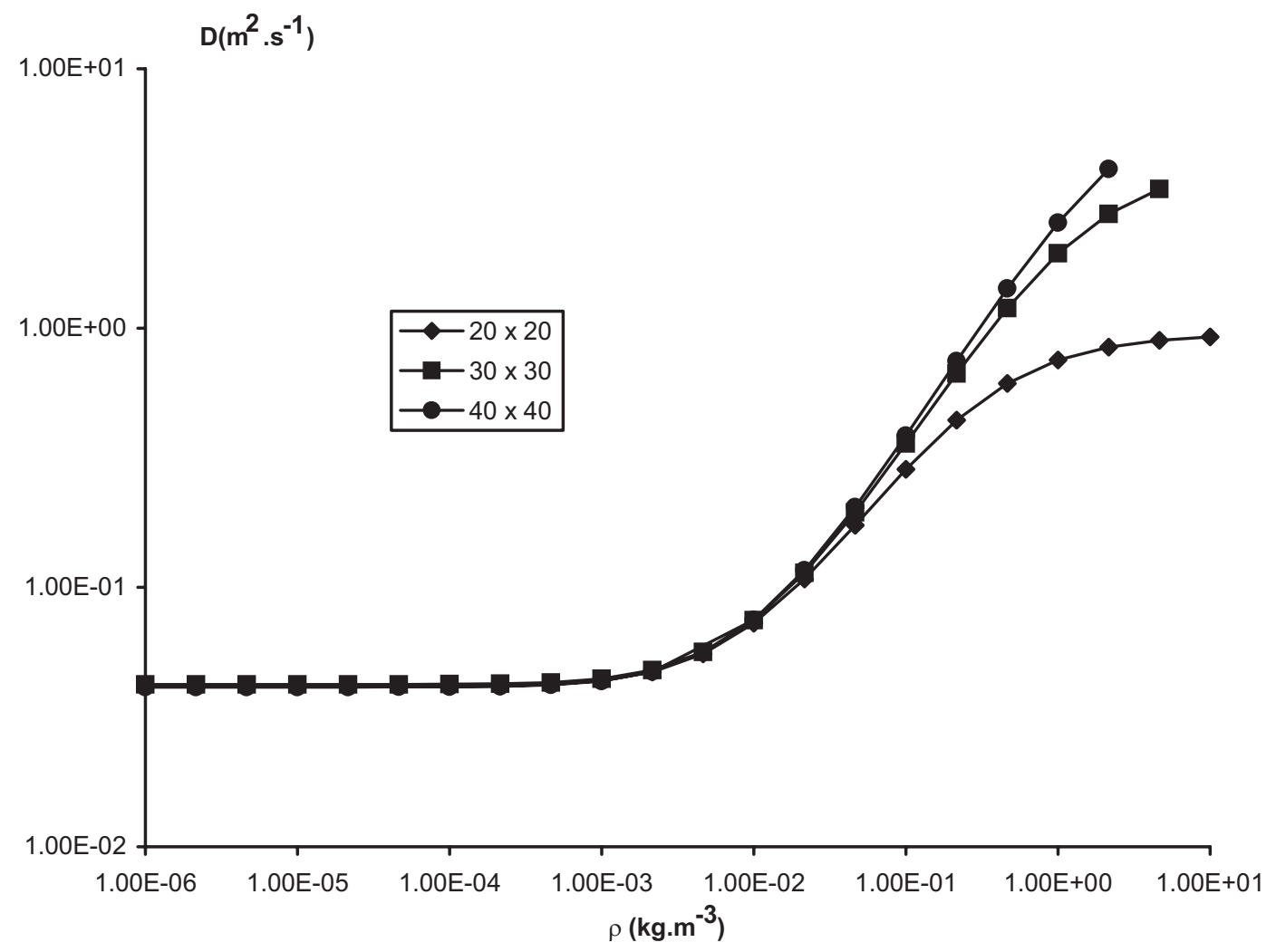

Figure 17. Coefficient $D$ as a function of gas density $\rho$ for various discretization parameters at $T=400 \mathrm{~K}$, for a cylindrical pore. The accommodation coefficient is $\sigma=0.6$ and the ES-BGK scheme has been used. 


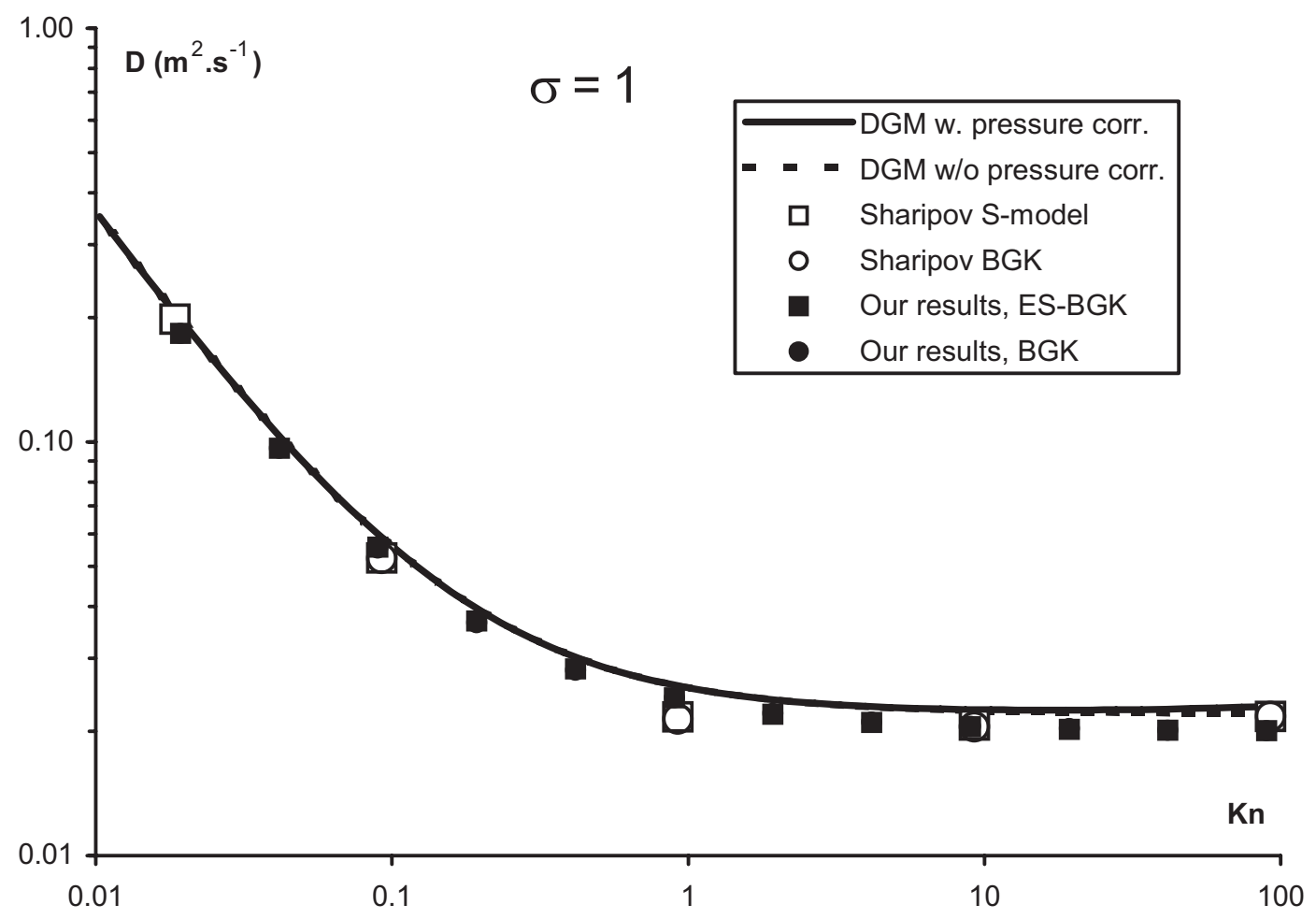

Figure 18. Coefficient $D$ as a function of Knudsen number $K n=\frac{\eta}{\rho D_{K, \text { ref }}}$ at $T=400$ $K$, for a cylindrical pore. The accommodation coefficient $\sigma$ is unity. 


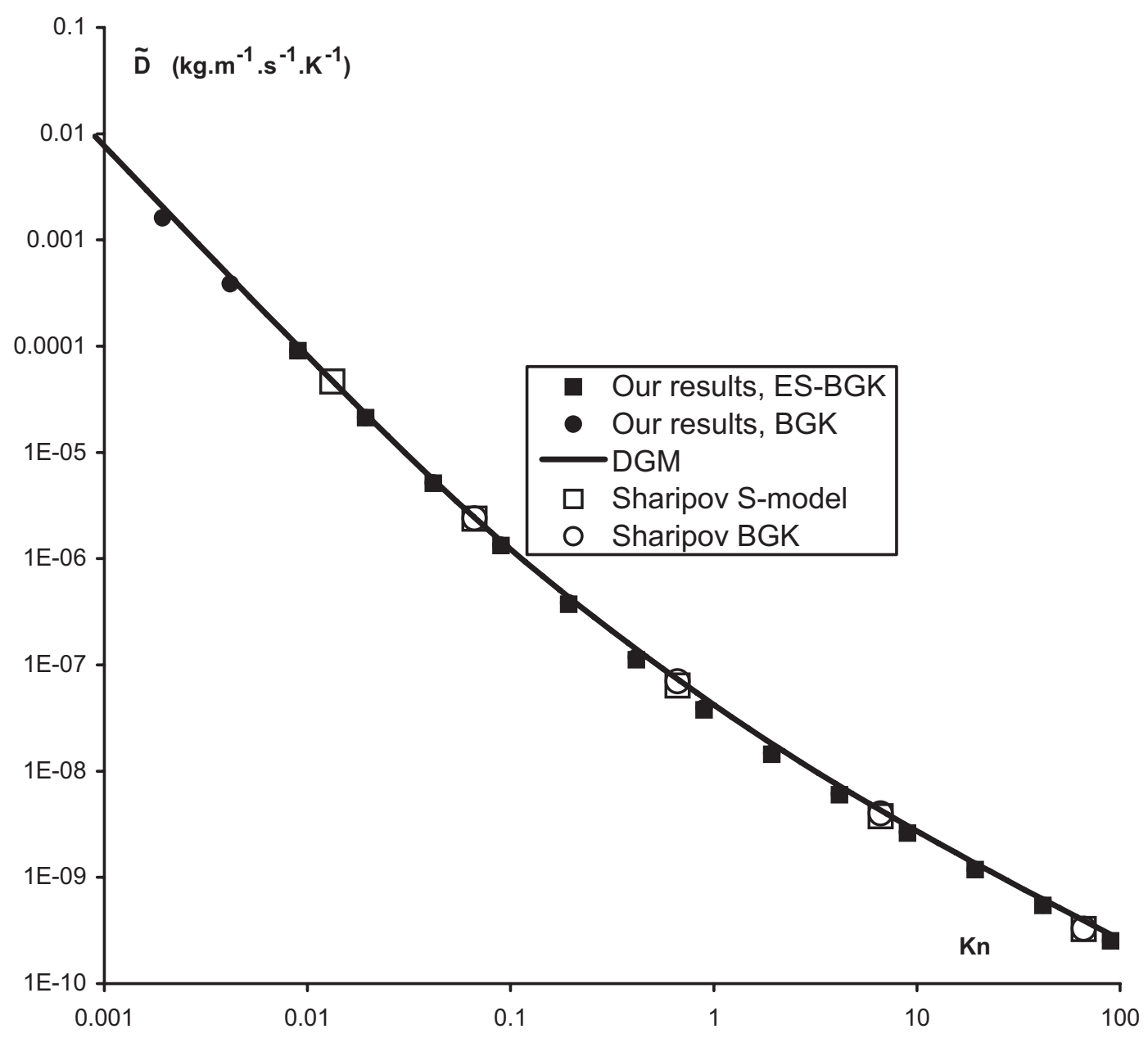

Figure 19. Coefficient $\tilde{D}$ as a function of Knudsen number $K n$ at $T=400 K$, for a cylindrical pore. The accommodation coefficient $\sigma$ is unity. 


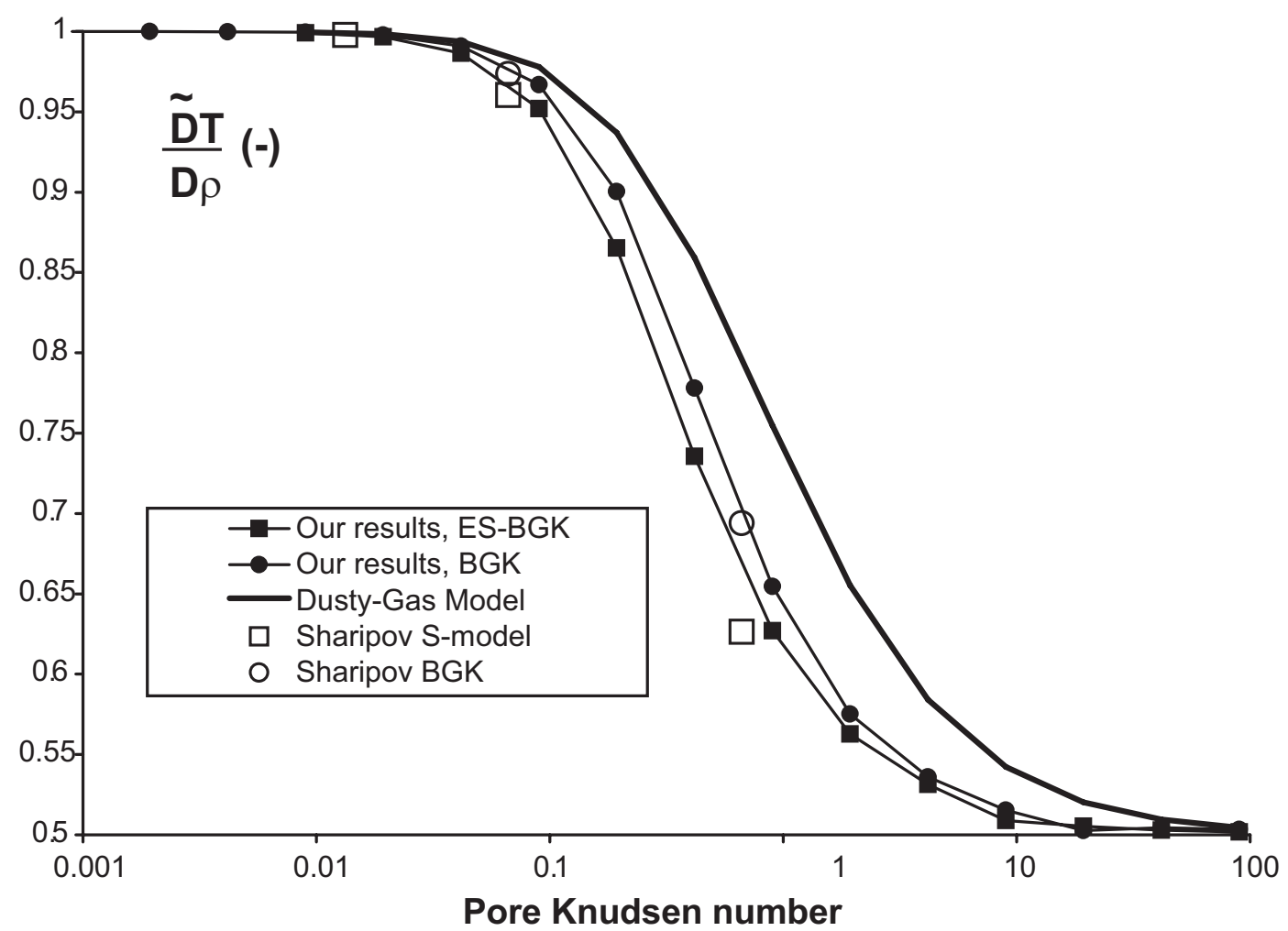

Figure 20. Coefficient ratio $\frac{\tilde{D} T}{D \rho}$ as a function of Knudsen number $K n$ at $T=400 K$ for a cylindrical pore. The accommodation coefficient $\sigma$ is unity. 


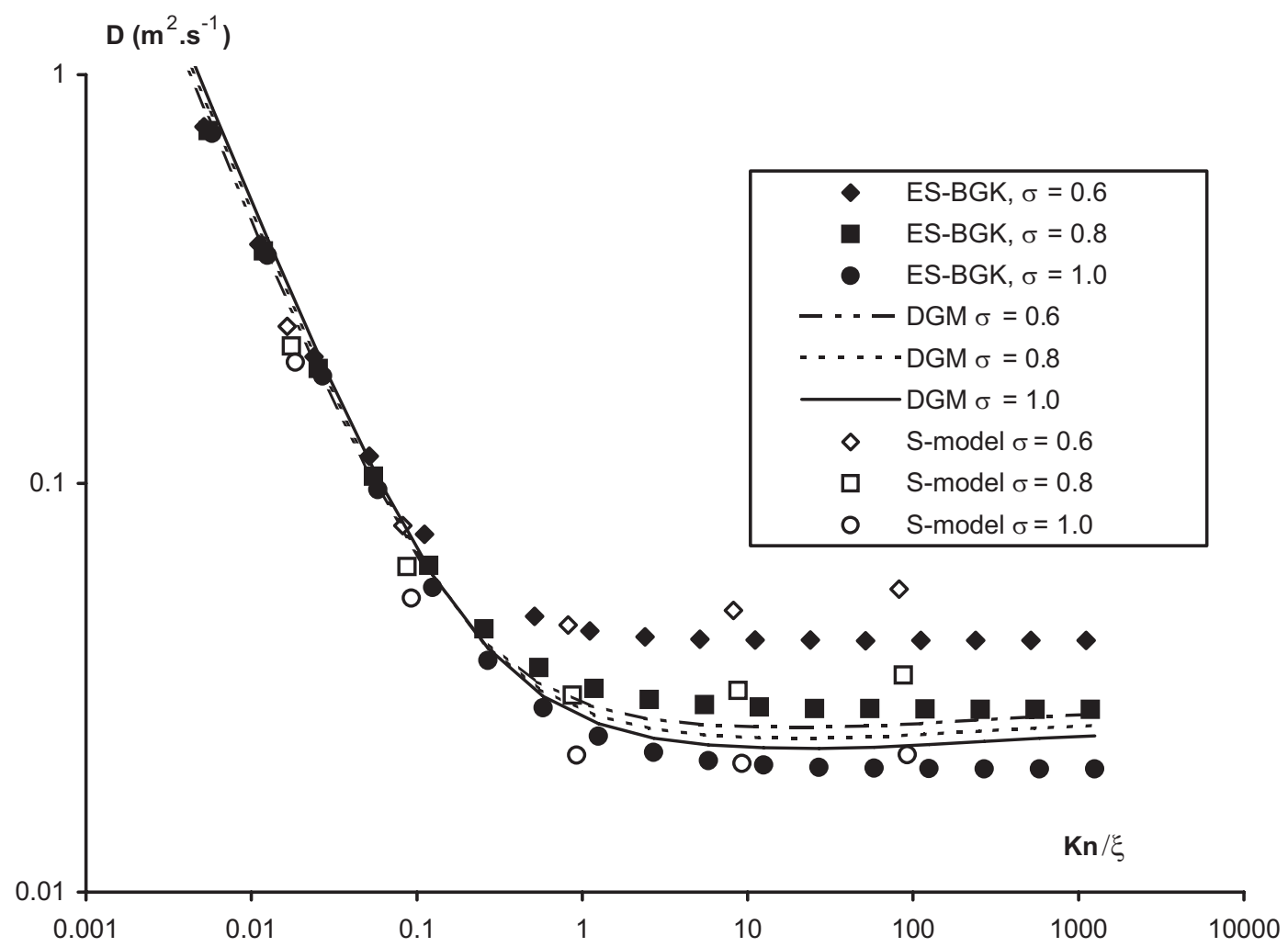

Figure 21. Coefficient $D$ as a function of scaled Knudsen number $K n / \xi$ at $T=400 K$ for a cylindrical pore, with various values of the accommodation coefficient $\sigma$. 


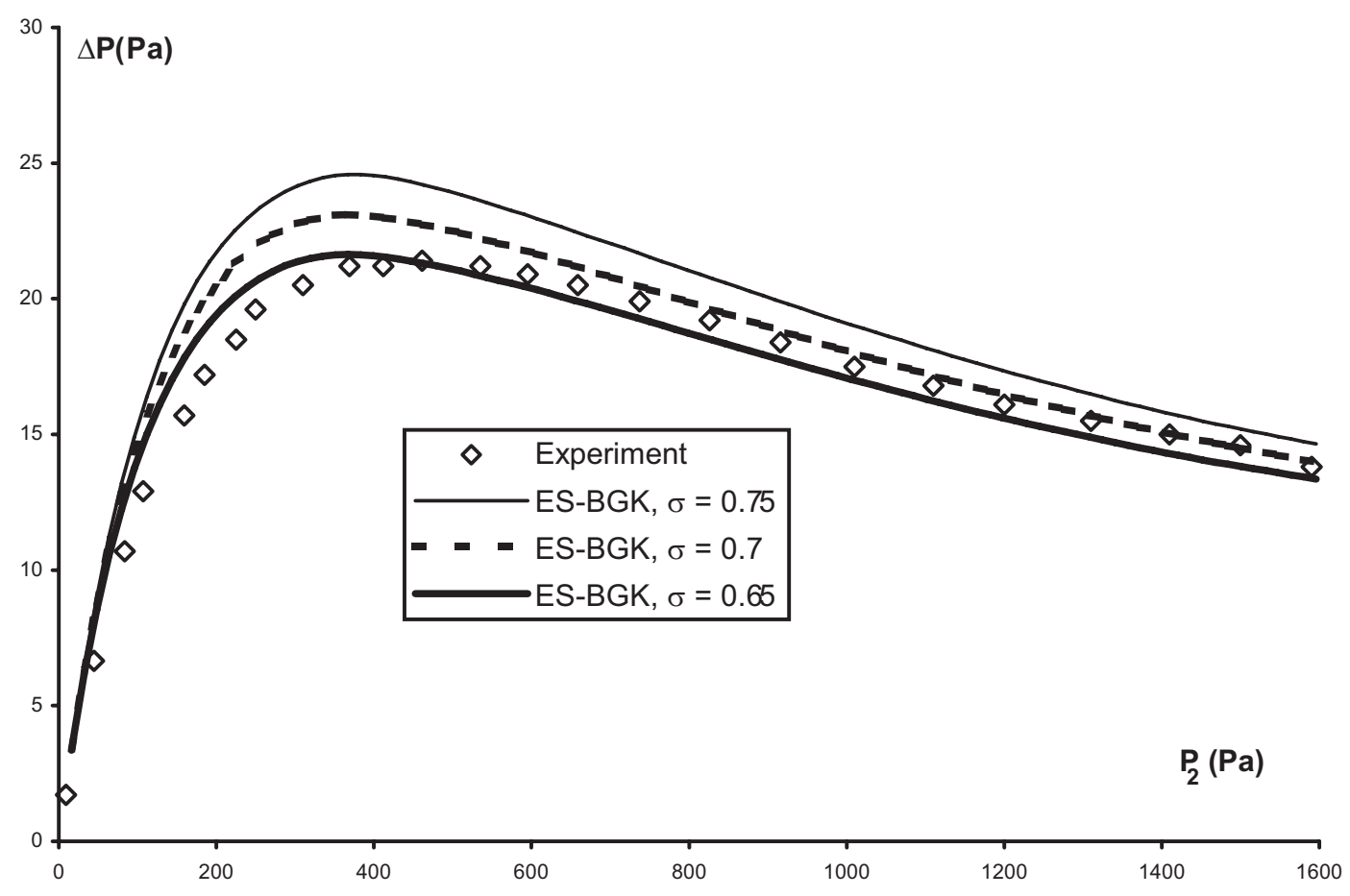

Figure 22. Computed vs. experimental curves of pressure drop in thermal transpiration experiment.

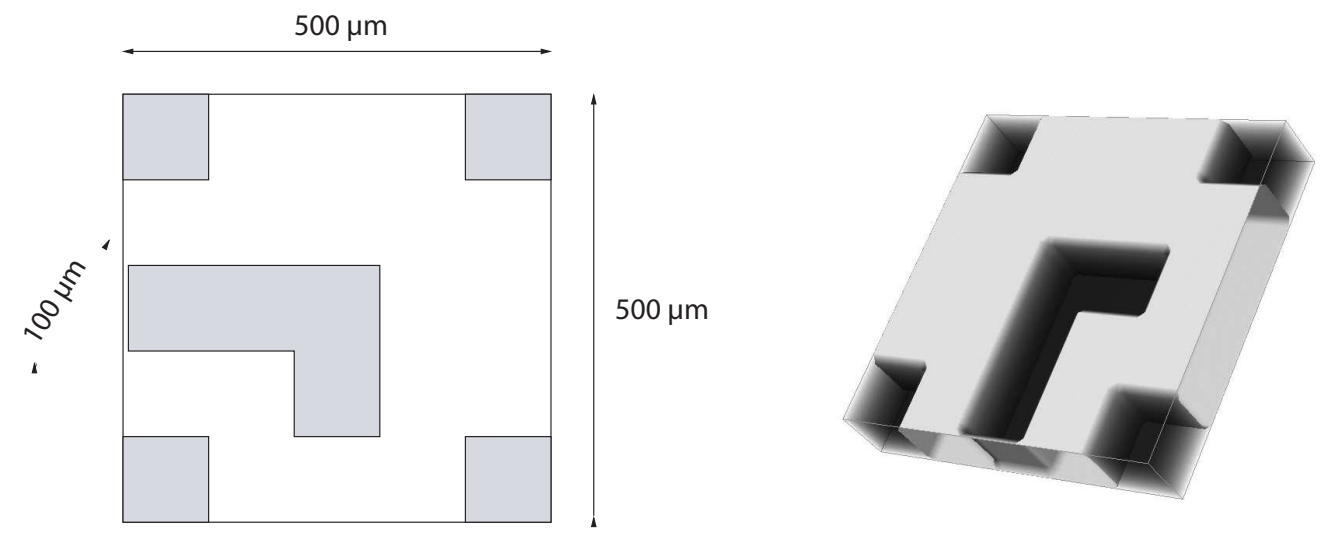

Figure 23. Visualization of the test porous medium unit cell $\mathrm{n}^{\circ} 1$. 


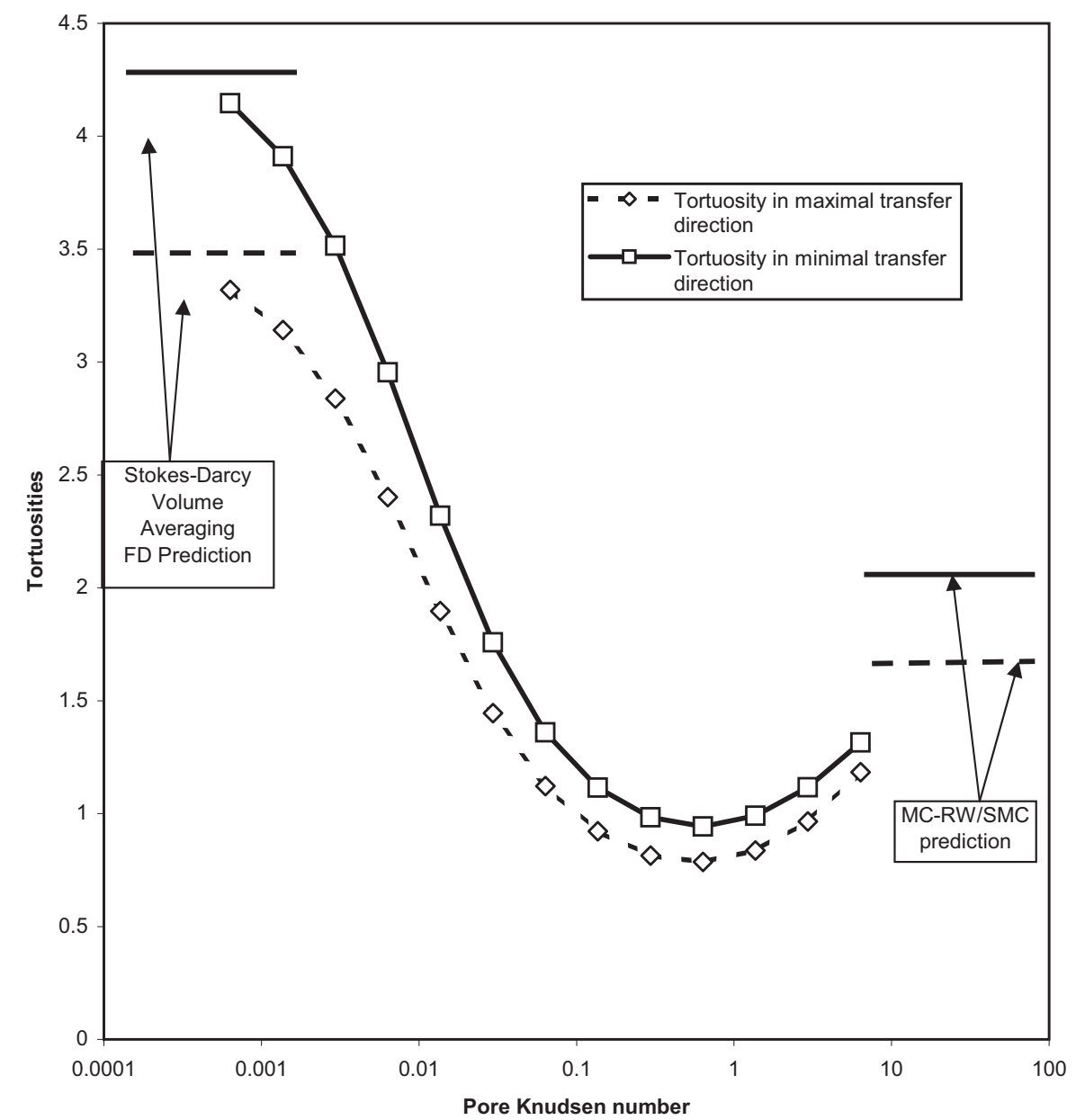

Figure 24. Tortuosity evaluation of test porous medium no.1 with respect to mass transfer : comparison of the presented code and of two independent estimations of the continuum limit (Volume Averaging FD) and of the rarefied limit (MC-RW/SMC). 


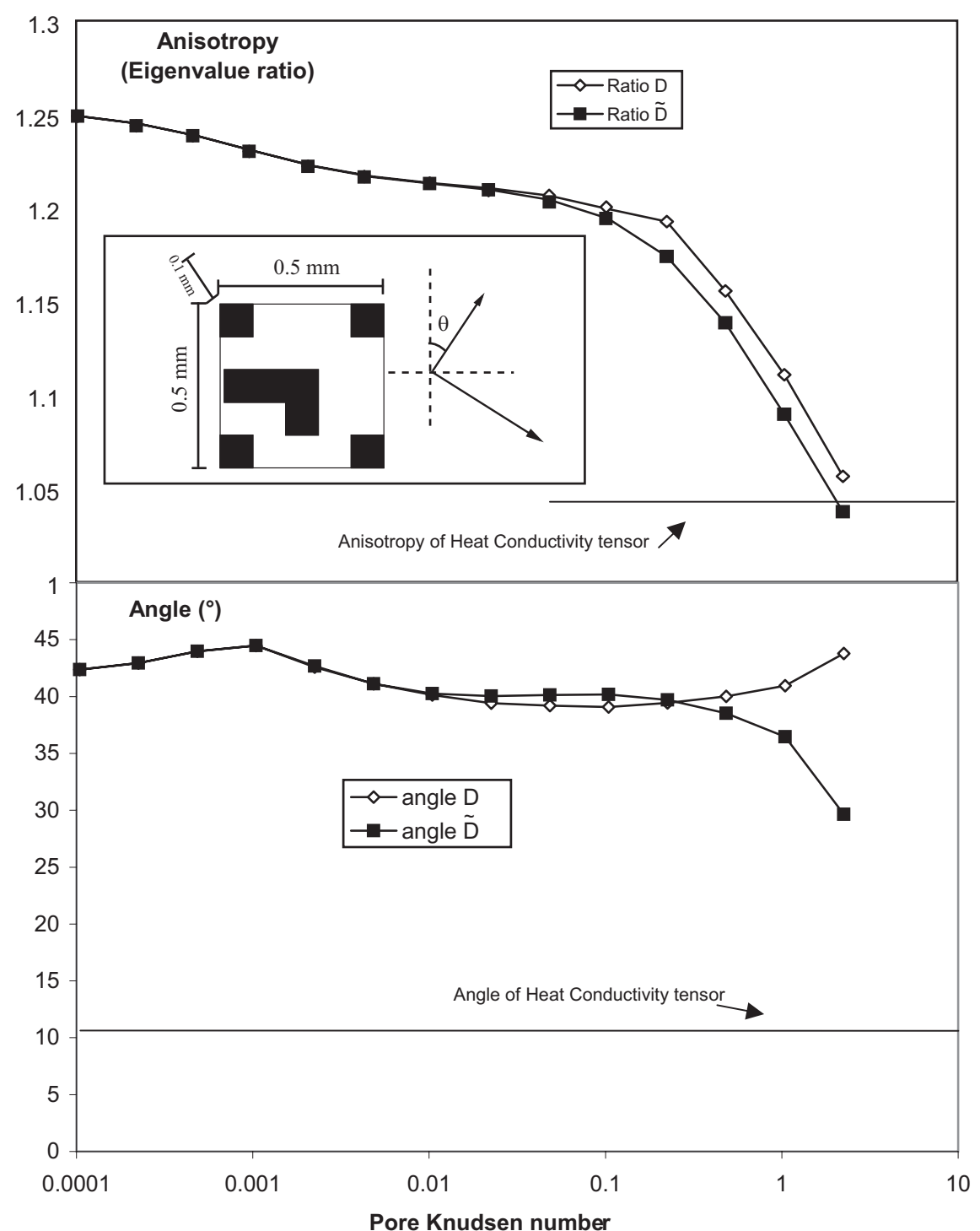

Figure 25. Angles and anisotropy ratios of $D$ and $\tilde{D}$ principal tensor components for porous medium $\mathrm{n}^{\circ} 1$ as a function of the pore Knudsen number. The values for the heat conductivity tensor are also provided for comparison. 

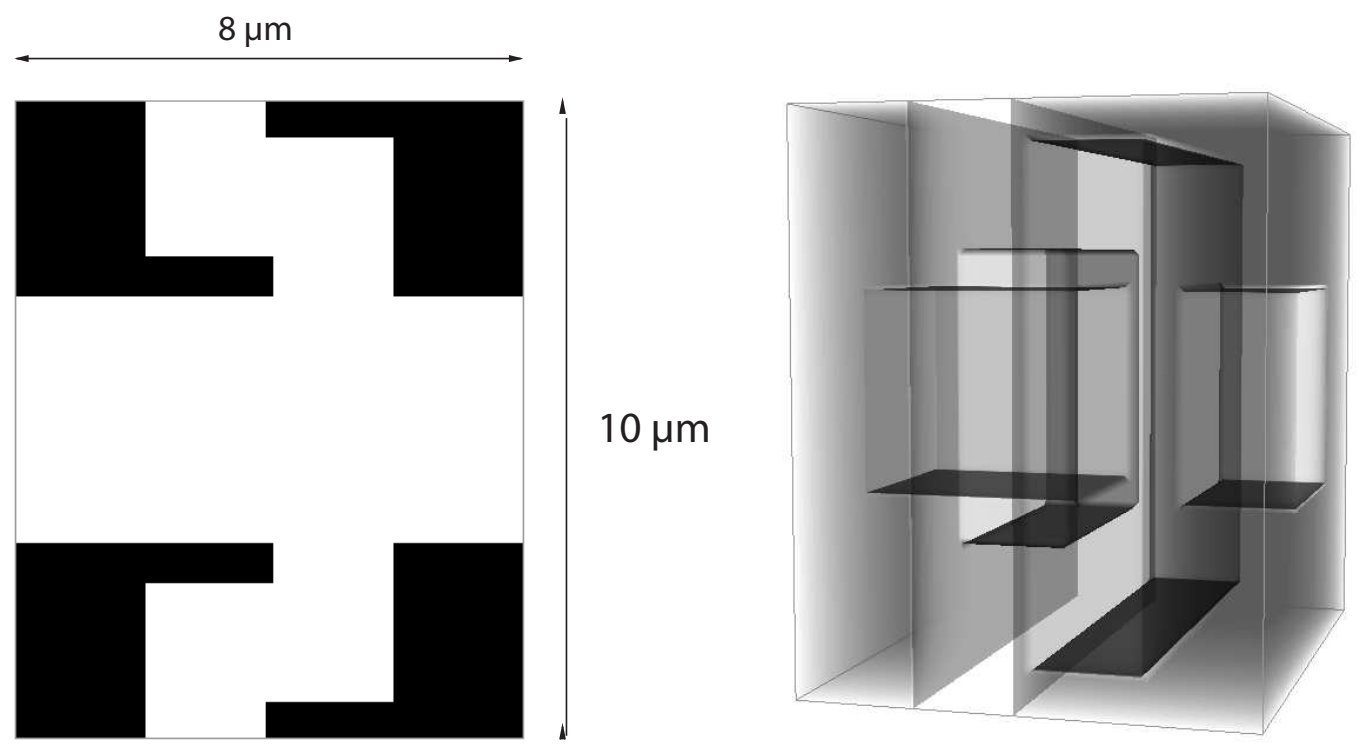

Figure 26. Visualization of the test porous medium unit cell $\mathrm{n}^{\circ} 2$. 


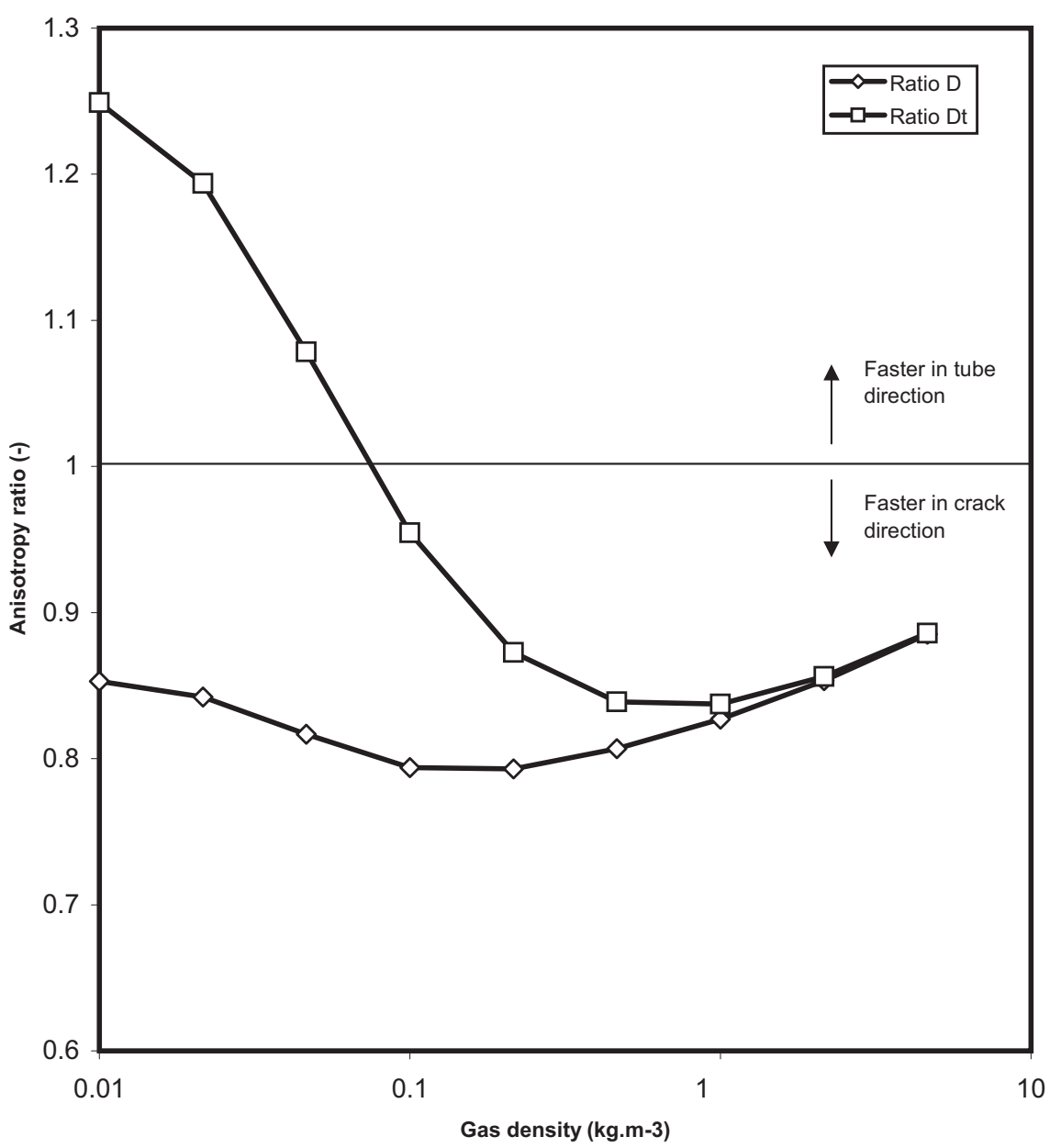

Figure 27. Anisotropy ratios of $D$ and $\tilde{D}$ principal tensor components for porous medium $\mathrm{n}^{\circ} 2$ as a function of gas density. 

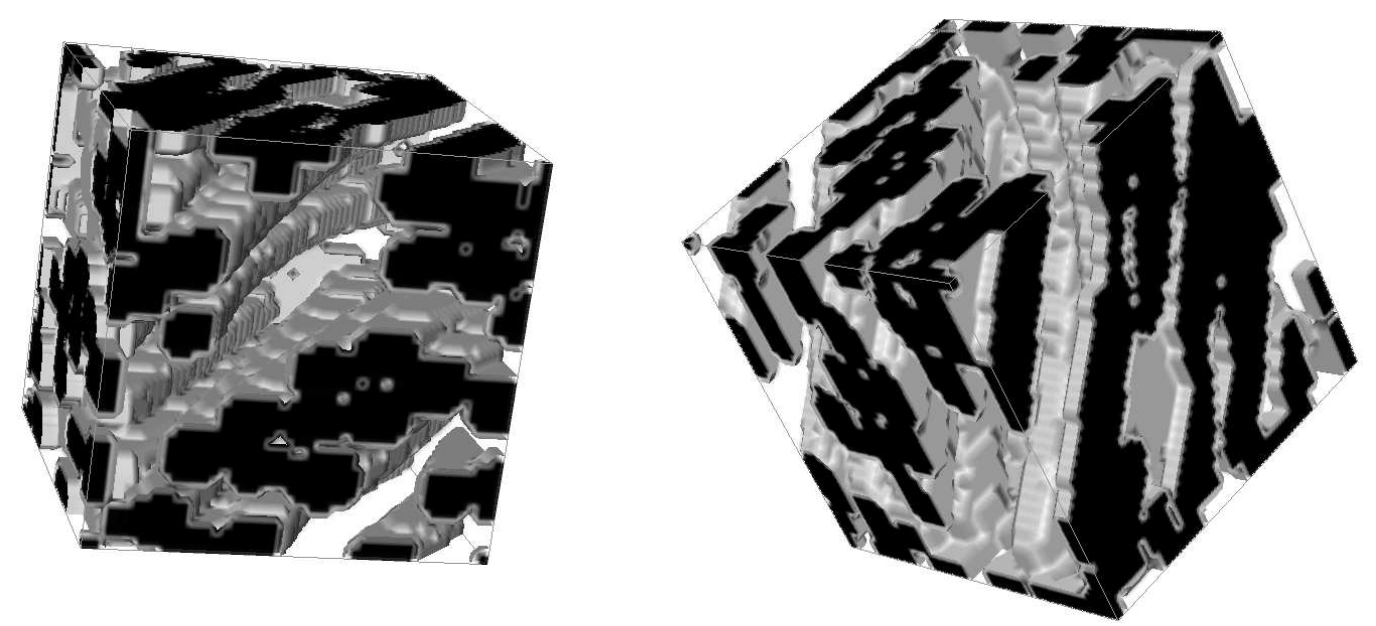

Figure 28. Visualizations of the test porous medium unit cell $\mathrm{n}^{\circ} 3$.
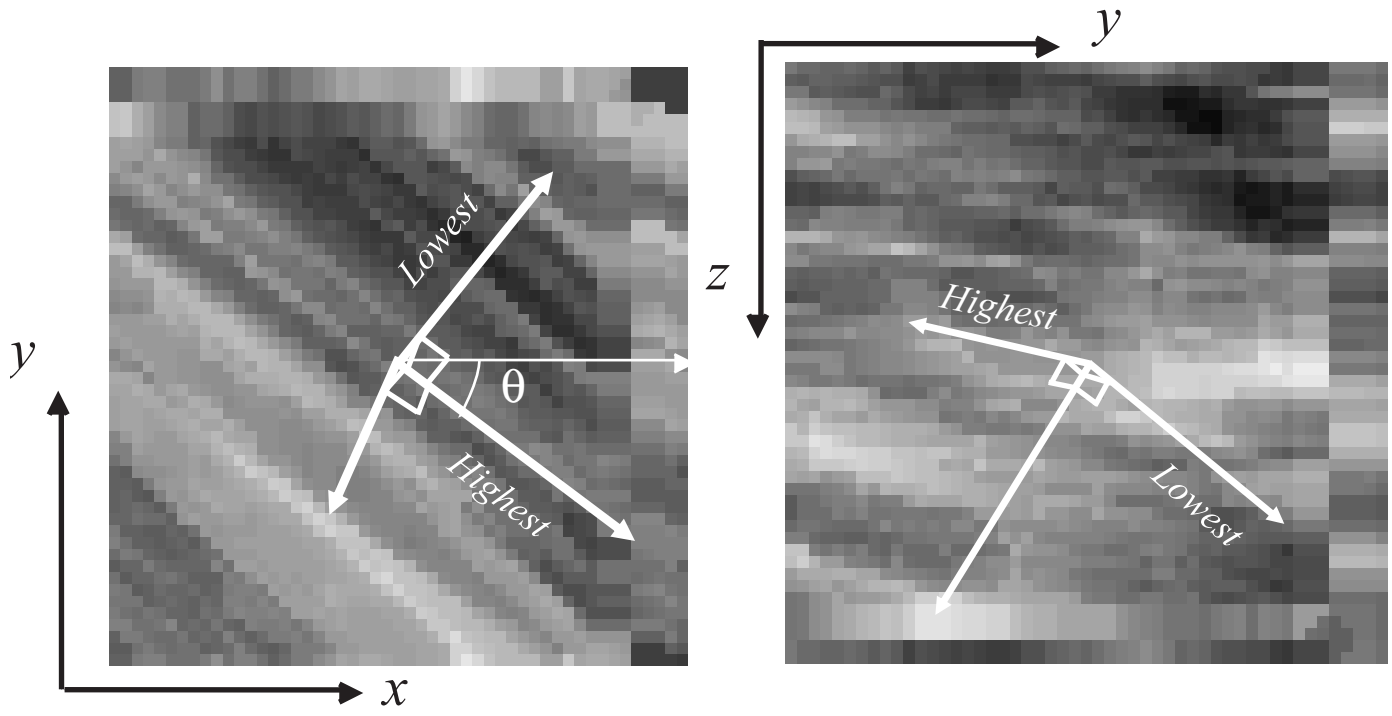

Figure 29. Visualizations of the principal tensor components for test porous medium unit cell $\mathrm{n}^{\circ} 3$ on $z$ and $x$ projections. 


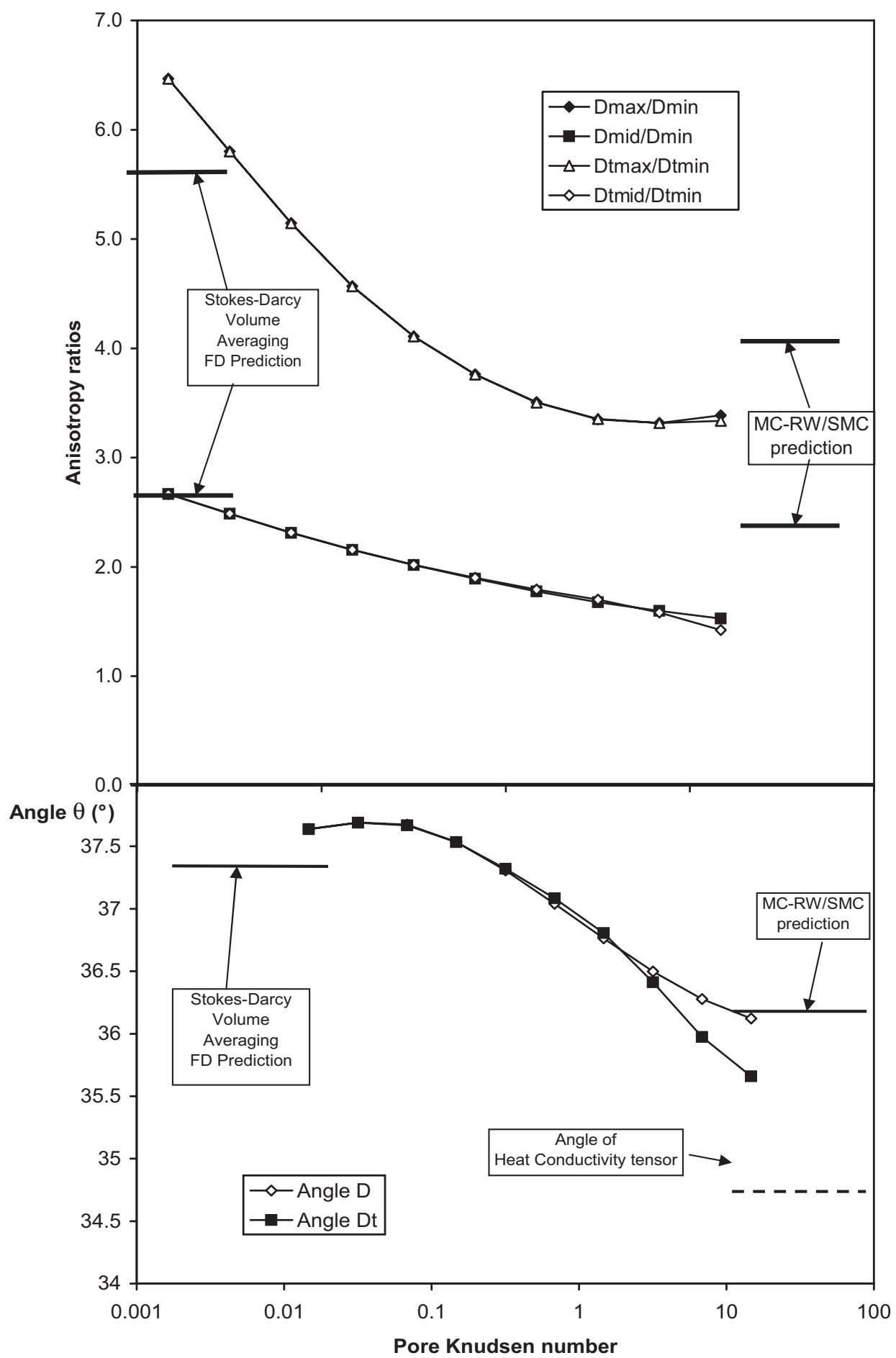

Figure 30. Anisotropy ratios of $D$ and $\tilde{D}$ principal tensor components for porous medium $\mathrm{n}^{\circ} 3$ as a function of gas density. The definition of angle $\theta$ refers to fig. 14 . 
Article_tt_part2_rev1.tex; 15/01/2009; $11: 35 ;$ p.48 